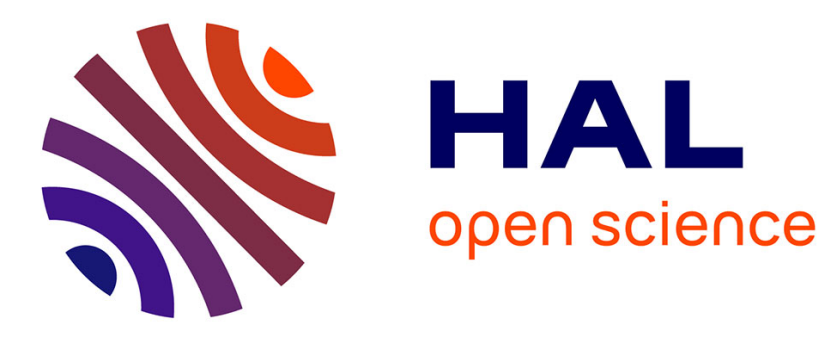

\title{
Nonlinear normal modes and band zones in granular chains with no pre-compression
}

K. R. Jayaprakash, Yuli Starosvetsky, Alexander F. Vakakis, Maxime Peeters, Gaetan Kerschen

\section{- To cite this version:}

K. R. Jayaprakash, Yuli Starosvetsky, Alexander F. Vakakis, Maxime Peeters, Gaetan Kerschen. Nonlinear normal modes and band zones in granular chains with no pre-compression. Nonlinear Dynamics, 2011, 63 (3), pp.359-385. 10.1007/s11071-010-9809-0 . hal-01510822

\section{HAL Id: hal-01510822 \\ https://hal.science/hal-01510822}

Submitted on 20 Apr 2017

HAL is a multi-disciplinary open access archive for the deposit and dissemination of scientific research documents, whether they are published or not. The documents may come from teaching and research institutions in France or abroad, or from public or private research centers.
L'archive ouverte pluridisciplinaire HAL, est destinée au dépôt et à la diffusion de documents scientifiques de niveau recherche, publiés ou non, émanant des établissements d'enseignement et de recherche français ou étrangers, des laboratoires publics ou privés.

\section{(c)(1)}

Distributed under a Creative Commons Attribution| 4.0 International License 


\title{
Nonlinear normal modes and band zones in granular chains with no pre-compression
}

\author{
K.R. Jayaprakash • Yuli Starosvetsky • \\ Alexander F. Vakakis • Maxime Peeters • \\ Gaetan Kerschen
}

\begin{abstract}
We study standing waves (nonlinear normal modes-NNMs) and band zones in finite granular chains composed of spherical granular beads in Hertzian contact, with fixed boundary conditions. Although these are homogeneous dynamical systems in the notation of Rosenberg (Adv. Appl. Mech. 9:155$242,1966)$, we show that the discontinuous nature of the dynamics leads to interesting effects such as separation between beads, NNMs that appear as traveling waves (these are characterized as pseudo-waves), and localization phenomena. In the limit of infinite extent, we study band zones, i.e., pass and stop bands in the frequency-energy plane of these dynamical sys-
\end{abstract}

K.R. Jayaprakash · Y. Starosvetsky · A.F. Vakakis

Department of Mechanical Science and Engineering,

University of Illinois at Urbana Champaign,

1206 W. Green Street, Urbana, IL 61822, USA

e-mail: kalkunt1@illinois.edu

Y. Starosvetsky

e-mail: staryuli@illinois.edu

A.F. Vakakis

e-mail: avakakis@illinois.edu

M. Peeters · G. Kerschen

Department of Aerospace and Mechanical Engineering, University of Liege, 1, Chemin des Chevreuils (B52/3),

4000 Liege, Belgium

M. Peeters

e-mail:m.peeters@ulg.ac.be

G. Kerschen

e-mail: g.kerschen@ulg.ac.be tems, and classify the essentially nonlinear responses that occur in these bands. Moreover, we show how the topologies of these bands significantly affect the forced dynamics of these granular media subject to narrowband excitations. This work provides a classification of the coherent (regular) intrinsic dynamics of one-dimensional homogeneous granular chains with no pre-compression, and provides a rigorous theoretical foundation for further systematic study of the dynamics of granular systems, e.g., the effects of disorders or clearances, discrete breathers, nonlinear localized modes, and high-frequency scattering by local disorders. Moreover, it contributes toward the design of granular media as shock protectors, and in the passive mitigation of transmission of unwanted disturbances.

Keywords Nonlinear normal modes - Granular media $\cdot$ Band gaps

\section{Introduction}

One defines nonlinear normal modes (NNMs) as synchronous periodic particular solutions of the nonlinear equations of motion of dynamical systems, keeping in mind the fact that the superposition principle is no longer valid in the nonlinear case. With such a restricted definition, a nonlinear generalization of the concept of normal mode of linear vibration theory is possible, and beginning with the works of Lyapunov 
[1] several attempts were made in this direction. Lyapunov's theorem proves the existence of $n$ synchronous periodic solutions (NNMs) in neighborhoods of stable equilibrium points of $n$ degrees-of-freedom (DOF) Hamiltonian systems whose linearized eigenfrequencies are not integrably related. The formulation and development of the theory of NNMs can be attributed to Rosenberg and his co-workers who developed general qualitative [2] and quantitative [3-6] techniques for analyzing NNMs in discrete conservative oscillators. Rosenberg considered $n$ DOF conservative oscillators and defined NNMs as vibrations-inunison, i.e. synchronous time-periodic motions during which all coordinates of the system vibrate equiperiodically (in a synchronous fashion), reaching their maximum and minimum values at the same instant of time. In linearizable systems with weak nonlinearities it is natural to suppose that NNMs are particular periodic solutions that, as the nonlinearities tend to zero, approach in limit the classical normal modes of the corresponding linearized systems. On the contrary, essentially nonlinear systems do not show such a behavior, since the NNMs in these systems are not necessarily extensions of normal modes of linear systems. Thus the NNMs in these systems may exceed in number the degrees-of-freedom of the system, and, in addition, certain of the NNMs may not have counterparts in linear theory. This is due to NNM bifurcations, which become exceedingly more complicated as the number of DOF of the systems increases and introduces new features, such as nonlinear mode localization, in the dynamics.

The discussion of NNMs in the literature has focused mainly on dynamical systems with smooth nonlinearities [1-8], although some works on systems with non-smooth nonlinearities have appeared [27-29]. In this work we will consider NNMs in homogeneous granular chains with no pre-compression, possessing essential (nonlinearizable) nonlinearities due to Hertzian contact interactions between beads [9]. In such strongly nonlinear systems we can extend the definition of NNM but we will need to introduce some modifications. For example, it will be shown that the condition of synchronicity is not valid for certain of the nonlinear modes of granular chains. We mention at this point that the dynamics of granular chains has been studied extensively both analytically and experimentally. Notably, in the works by Nesterenko [9], Daraio et al. [10, 11], Sen et al. [12],
Nesterenko et al. [13] and Herbold et al. [14] different aspects of disturbance transmission in homogeneous and disordered granular chains were studied.

Granular media is a highly complex and distinct class of dynamical systems. The dynamics of these media is highly tunable, and depending on the applied pre-compression it can be either strongly or weakly nonlinear and smooth or non-smooth. Indeed, for strong pre-compression the dynamics of granular media is weakly nonlinear, whereas when no precompression is applied the dynamics is strongly nonlinear (in fact, not even linearizable) and separation between beads is possible (in the absence of compressive forces that keep neighboring beads in contact). Hence, the dynamics of granular chains with no (or sufficiently weak) pre-compression is either smooth - when neighboring beads are in contactor non-smooth when separation between beads takes place and collisions between beads take place. This forms an added source of strong nonlinearity in the granular medium, i.e., in addition to the strongly nonlinear Hertzian law interaction between beads in contact.

In this work we will consider one-dimensional homogeneous granular media in their essentially nonlinear limit of absence of pre-compression between beads. In this case the force $(F)$ vs. displacement $(d)$ law governing the Hertzian interaction between neighboring spherical beads is highly nonlinear:

$F= \begin{cases}k d^{3 / 2}, & d \geq 0 \\ 0, & d<0\end{cases}$

since it lacks a linear term in compression and is zero in extension (when bead separation occurs); in the above relation, $k$ is the nonlinear coefficient. Such a granular system does not support oscillations of individual beads about zero mean positions, but rather their oscillations are highly asymmetric and nonsynchronous. Such systems have been characterized by Nesterenko [9] as "acoustic vacua" or "sonic vacuum," meaning sound propagation is impossible in these systems. More precisely, it implies the impossibility for linear waves described by standard wave equation to propagate in them. It follows that the analysis of the dynamics requires the application of strongly nonlinear methods that are individually tailored for this class of systems since linearized or weakly nonlinear methods are not applicable. 
Our study of standing waves (NNMs) in onedimensional granular media will be structured as follows. In Sect. 2, we initiate our analysis by considering a two-bead system with fixed boundary conditions, and study NNMs by numerical Poincaré maps and analytical techniques in terms of hypergeometric functions [15]. In Sect. 3 we will extend our analysis to three-bead granular systems, and compute the corresponding NNMs by numerical methods. In Sect. 4 we will focus on higher-dimensional (but finite-dimensional) systems and consider exclusively two specific classes of NNMs, namely the in-phase and out-of-phase ones since, as we will show, these form the boundaries of the frequency-energy band within which all other NNMs are realized. In Sect. 5 we will study the dynamics of infinite-dimensional homogeneous granular chains, and define propagation (pass) and attenuation (stop) bands in the frequencyenergy plane, within which disturbances either propagate or attenuate, respectively, as they are transmitted though these media. The effect of these bands on the forced dynamics of high-dimensional systems will be studied in Sect. 6, where numerical integrations will confirm the theoretical predictions regarding the role of the pass and stop bands on the dynamics. Some concluding remarks and a summary of the main findings will be provided in Sect. 7 .

\section{Two-bead granular system}

The two-bead granular system considered is depicted in Fig. 1. The system is homogeneous in the sense that the two spherical beads have identical geometric and material properties. Moreover, the beads are placed between rigid boundaries (walls) with no gaps existing between them or with the walls when they are in their trivial equilibrium positions. The system is considered without any pre-compression, and thus it exhibits strong (essential) stiffness nonlinearity and potentially non-smooth effects. Furthermore, no dissipative effects are yet considered in this study, but weak dissipative forces in the bead interactions will be considered later, in our computational study of the forced dynamics of high-dimensional granular media carried out in Sect. 6. Assuming Hertzian contact interaction between beads and between the end beads and the rigid walls, the kinetic and potential energies of the two-
Fig. 1 The two-bead system

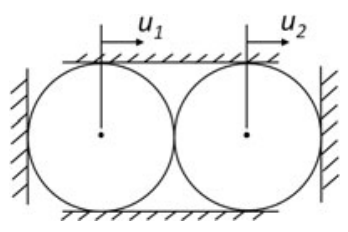

bead system are defined as follows:

$$
\begin{aligned}
T= & \frac{1}{2} m\left(\dot{u}_{1}^{2}+\dot{u}_{2}^{2}\right) \\
U= & \frac{2}{5} \frac{E(2 R)^{1 / 2}}{3\left(1-\mu^{2}\right)} \\
& \times\left[\left(-u_{1}\right)_{+}^{5 / 2}+\left(u_{2}\right)_{+}^{5 / 2}+\left(u_{1}-u_{2}\right)_{+}^{5 / 2}\right]
\end{aligned}
$$

where, $u_{i}$ is the displacement of the $i$ th bead, $R$ denotes radius, $E$ elastic modulus, $\mu$ Poisson's ratio, $\rho$ mass density, and $m$ mass of each bead; the (+) subscript in the expressions in (1) is used to emphasize that the bracketed term is non-zero only if the term inside the bracket is positive and zero otherwise. The equations of motion can be derived from Lagrange's equation as follows:

$\ddot{u}_{1}=A\left[\left(-u_{1}\right)_{+}^{3 / 2}-\left(u_{1}-u_{2}\right)_{+}^{3 / 2}\right]$
$\ddot{u}_{2}=A\left[-\left(u_{2}\right)_{+}^{3 / 2}+\left(u_{1}-u_{2}\right)_{+}^{3 / 2}\right]$

where $A=E(2 R)^{1 / 2} /\left[3 m\left(1-\mu^{2}\right)\right]$.

The displacements are non-dimensionalized by means of the following normalizations:

$$
\begin{aligned}
X & =\frac{u}{R}, \quad \tau=\frac{t}{2 R / C}\left(\frac{\pi\left(1-\mu^{2}\right)}{\sqrt{2}}{ }^{-1 / 2}\right. \\
C & =\overline{\left(\frac{E}{\rho}\right.}
\end{aligned}
$$

so the equations of motion are placed in the following normalized form:

$$
\begin{aligned}
& X_{1}^{\prime \prime}=\left(-X_{1}\right)_{+}^{3 / 2}-\left(X_{1}-X_{2}\right)_{+}^{3 / 2} \\
& X_{2}^{\prime \prime}=-\left(X_{2}\right)_{+}^{3 / 2}+\left(X_{1}-X_{2}\right)_{+}^{3 / 2}
\end{aligned}
$$

where $X_{i}=X_{i}(\tau)$ in the terms of the normalized time $\tau$, and prime denotes differentiation with respect to $\tau$. System (3) will be employed in the following analysis. We re-emphasize at this point that the dynamics of system (3) is not only essentially nonlin- 


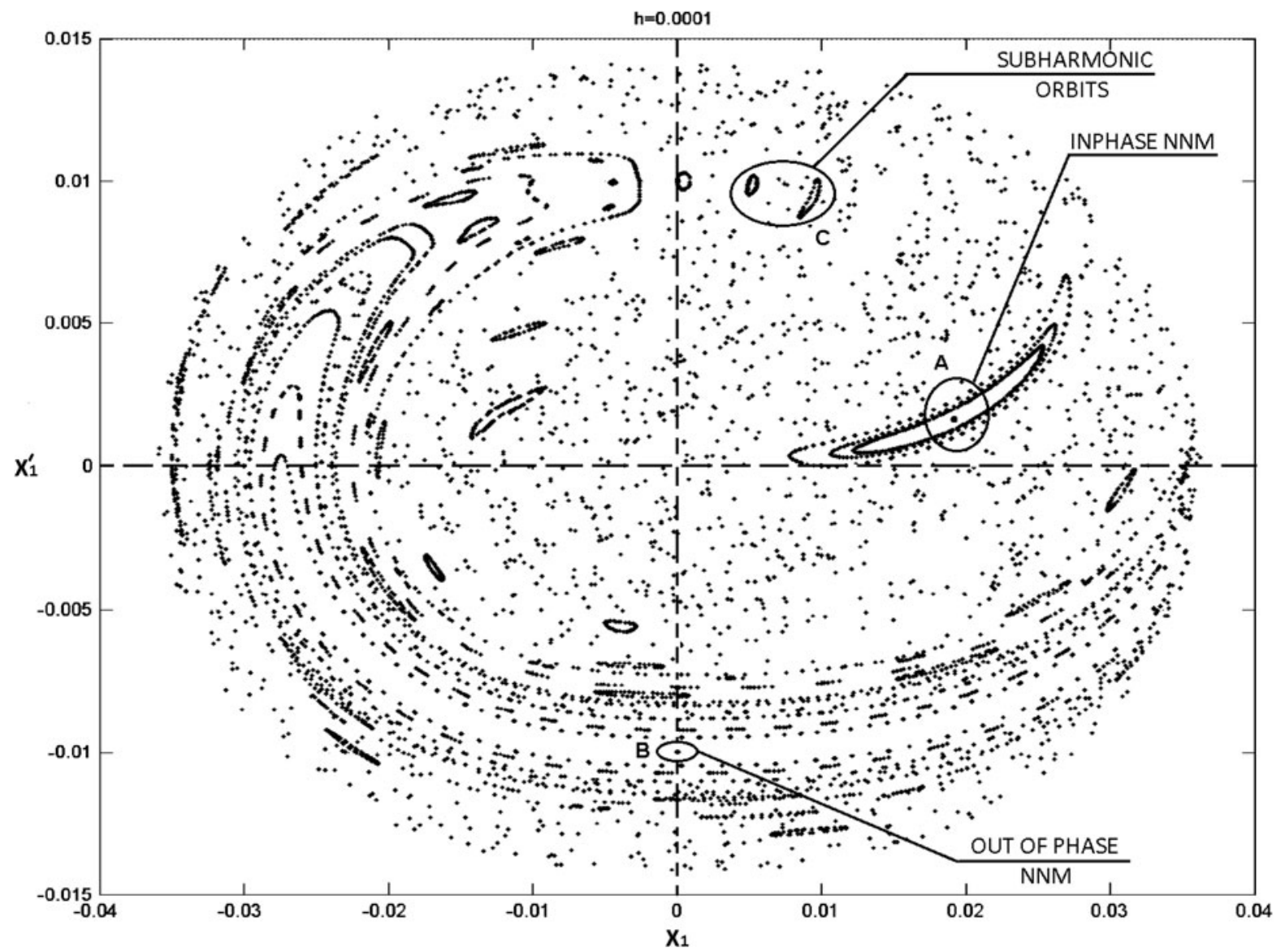

Fig. 2 Poincaré map of the global dynamics of the two-bead system for $h=0.0001$

ear (as the stiffness terms do not possess linear components), but, in addition, they are non-smooth. The loss of smoothness is due to the fact that the interaction force due to (compressive) Hertzian contact vanishes when the center distance between the two beads exceeds a length equal to twice the radius. From the above, we expect the dynamics to be highly complex.

Since the system possesses two degrees-of-freedom and is conservative, it is possible to analyze its global dynamics in terms of numerical Poincaré maps. Indeed, a nonlinear normal mode (NNM) of this system is defined as a time-periodic oscillation where the bead oscillations possess identical frequencies but are not necessarily synchronous. Moreover, whether the dynamics is smooth or non-smooth, a NNM should be depicted as a (modal) curve in the configuration plane. System (3) possesses a four-dimensional phase space, but owing to energy conservation (since no dissipative effects considered),

$$
\begin{aligned}
E\left(X_{1}, X_{1}^{\prime}, X_{2}, X_{2}^{\prime}\right) \equiv & (1 / 2)\left(X_{1}^{\prime 2}+X_{2}^{\prime 2}\right) \\
& +(2 / 5)\left[\left(-X_{1}\right)_{+}^{5 / 2}+\left(X_{2}\right)_{+}^{5 / 2}\right. \\
& \left.+\left(X_{1}-X_{2}\right)_{+}^{5 / 2}\right]=h
\end{aligned}
$$

the dimensionality can be reduced by one, and the dynamics can be restricted to a three-dimensional isoenergetic manifold. Intersecting the isoenergetic flow by a two-dimensional cut section defined as $T$ : $\left\{X_{2}=0\right\}$, we obtain a two-dimensional Poincaré map $P: \Sigma \rightarrow \Sigma$, where the Poincaré section $\Sigma$ is defined as $\Sigma=\left\{X_{2}=0, X_{2}^{\prime}>0\right\} \cap\left\{E\left(X_{1}, X_{1}^{\prime}, X_{2}, X_{2}^{\prime}\right)=h\right\}$ and depicts the global nonlinear dynamics of the system on the cut section that is now parameterized by $\left(X_{1}, X_{1}^{\prime}\right)$. In Fig. 2 we depict the numerical map at the energy level $h=0.0001$. Three types of periodic solutions are detected, namely an in-phase NNM, an 

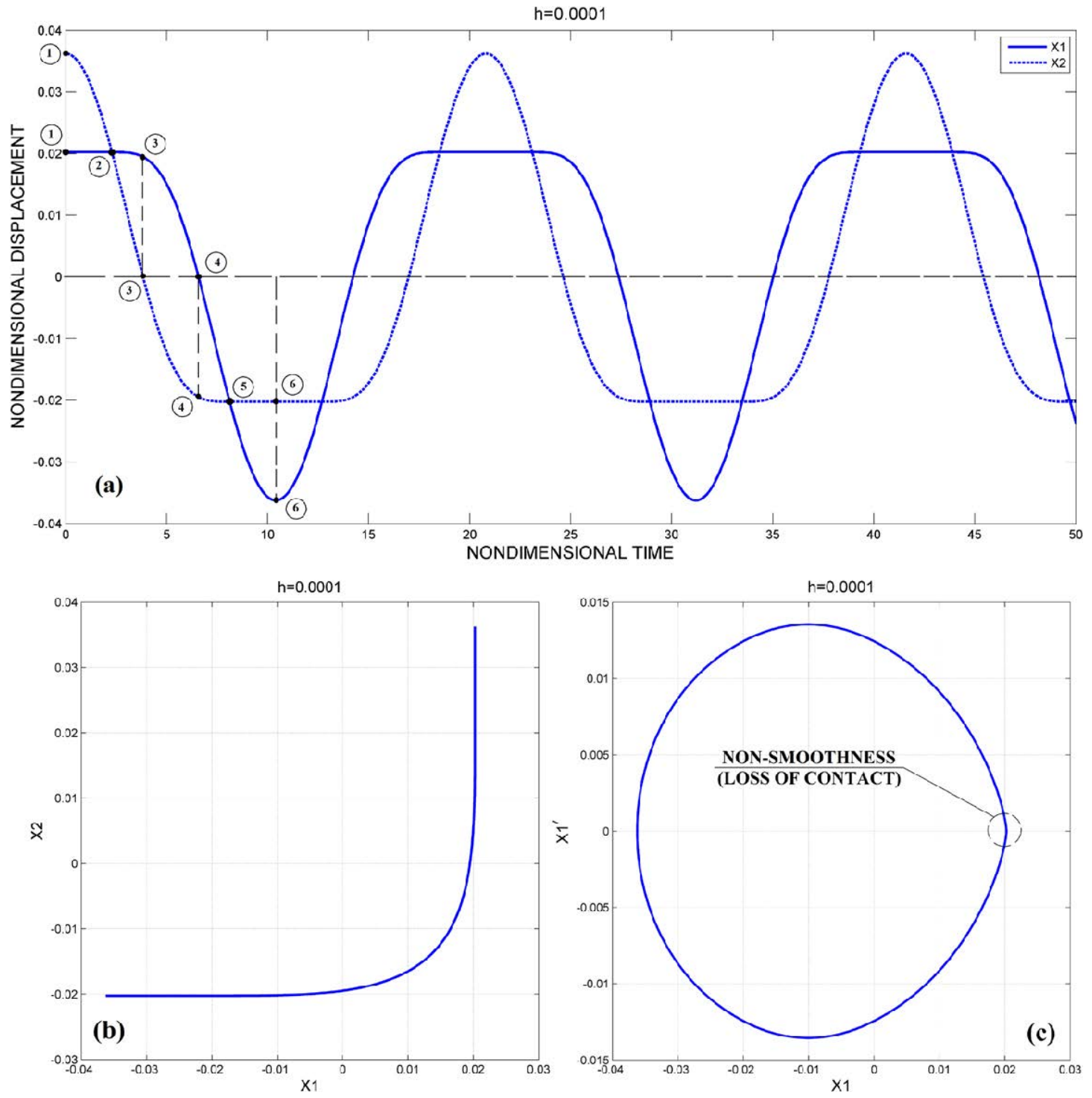

Fig. 3 The in-phase NNM for the two-bead granular system and $h=0.0001$ : (a) time series, (b) modal curve in the configuration plane, (c) depiction in a projection of the phase plane

out-of-phase NNM and numerous subharmonic orbits. We note that the out-of-phase NNM is a synchronous oscillation and lies on the $X_{1}$ axis on the cut section, whereas the in-phase NNM is offset from that axis and is an asynchronous oscillation. Moreover, the two modes appear to be stable and, hence, physically realizable.

In Fig. 3, we depict the in-phase NNM for the energy level $h=0.0001$ (region A of the Poincaré plot in Fig. 2). From the time series in Fig. 3a, we note that the two beads pass through their equilibrium posi- tions at different instants of time. Hence, such a mode does not conform to the traditional definition of NNM as proposed by Rosenberg [3]; however, by extending the definition to account for non-synchronicity in the bead oscillation, we may classify this time-periodic motion as in-phase NNM. The energy exchanges between beads for that mode are of particular interest. Referring to Fig. 3a, at point 1 the entire energy of the system is elastic due to the interaction of the second bead with the wall, whereas the first bead is motionless but offset from its equilibrium position. As 
time progresses, at point 2 the beads start interacting with each other, and at that time instant the first bead collides with the second. Proceeding to point 3 , the second bead loses contact with the wall as it passes through its equilibrium position. Once the first bead passes through its equilibrium position at point 4 , it starts interacting with the rigid wall and at that point the second bead has transferred almost all of its en- ergy to the first bead and is about to get stationary. At point 5, the second bead loses all its energy, becomes stationary but offset from its equilibrium position, and the entire process repeats itself. It is interesting to note that from point 2 to 5 , both beads remain in contact with each other, and, as explained previously, the second bead loses contact with the wall whereas the first bead gains contact with it. Beyond
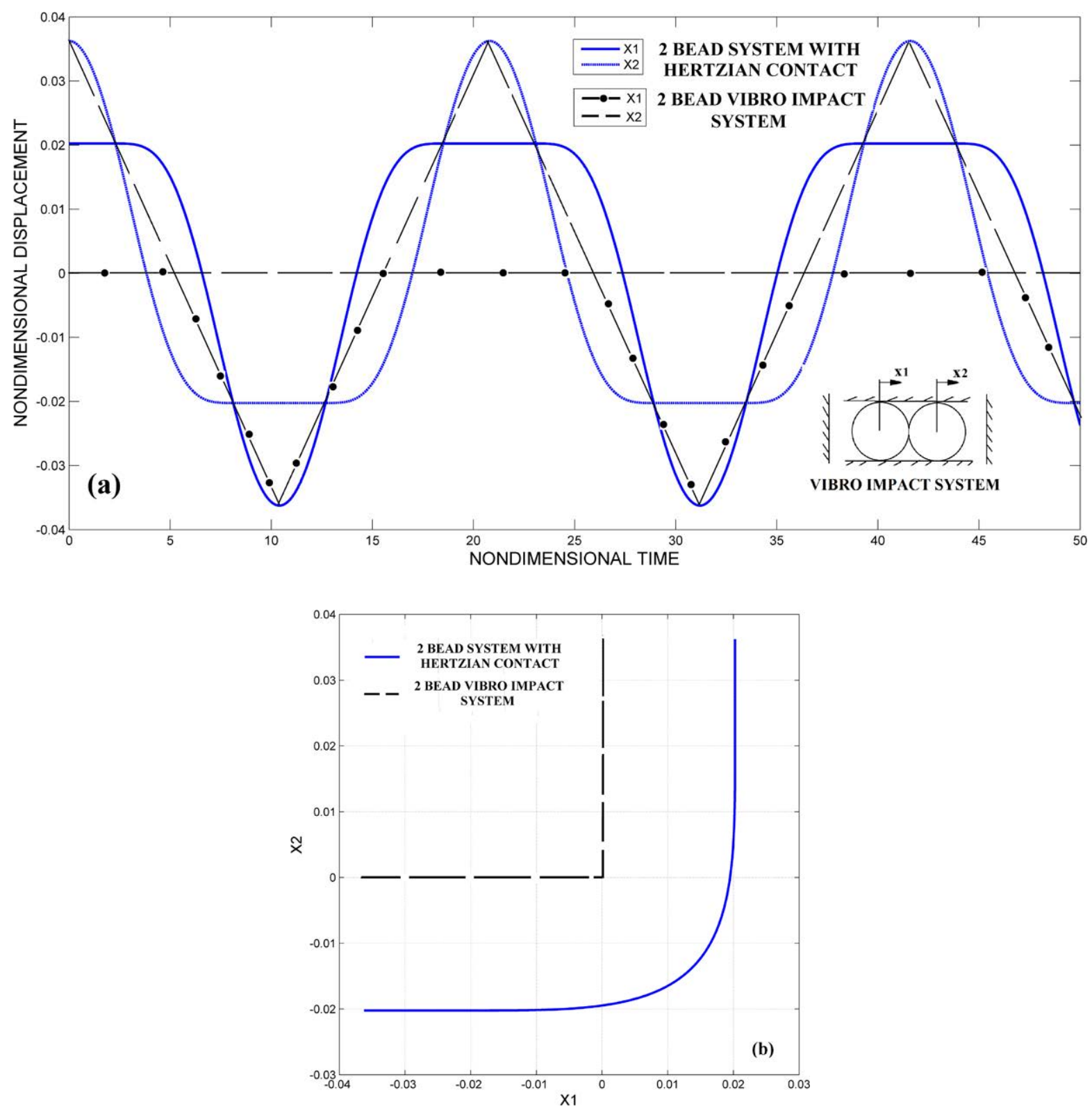

Fig. 4 Comparing the dynamics of the two-bead granular system to the dynamics of the corresponding vibro-impacting one: (a) time series, (b) modal curve in the configuration plane 
point 5 , the first bead interacts with the wall and subsequently loses contact with the second bead and the complete energy of the system is in form of elastic potential energy due to the interaction of the first bead and the wall (at point 6). Hence, complete exchange of energy between the two beads occurs, and the inphase NNM can be represented by the time-delayed relation $X_{1}(\tau)=-X_{2}(\tau-T / 2)$, where $T$ is the period of the in-phase NNM. The previous discussion highlights the complex and highly asymmetric oscillation of the granular system when it oscillates in this in-phase periodic motion.

The non-smooth character of the in-phase NNM can be inferred from the depiction of the dynamics in the projection of the phase plane (cf. Fig. 3c), where a discontinuity in slope can be readily noted. This happens at the time instant when one of the beads loses contact with the wall and the other stands stationary. It follows that non-smooth effects in the oscillation are associated with 'silent' periods of bead responses, i.e., with phases of the motion where a bead is stationary at an offset position from the zero equilibrium. Moreover, the depiction of the modal curve in the configuration plane in Fig. 3b confirms our earlier observation regarding the non-synchronicity of the bead oscillations since they cross their equilibrium points at different instants of time.

Finally, a comparative study of the dynamics of the two-bead granular system to the dynamics of a twoDOF vibro-impacting system (corresponding to perfectly rigid beads and purely elastic collisions between them and the wall) is performed in Fig. 4. The comparison is purely qualitative and thus we have attributed any energy to neither of the Hertzian or vibro impact systems. Due to the Hertzian contact law, deformation of beads during collisions occurs, and as a result the time series is smooth. By contrast, in the vibroimpacting system bead deformation does not occur, and so there exist non-smooth transitions between different phases of the dynamics. Still, the vibro-impact in-phase NNM could be considered as a generating solution to develop asymptotic approximations for the in-phase NNM of the granular medium in the limit of small bead deformations.

Focusing now on the out-of-phase NNM (region B in the Poincaré map in Fig. 2), in Fig. 5a we depict the corresponding responses of the two beads. In this case neither of the two beads reaches stationarity after separation, so no 'silent' regions exist, and the dynamics is smooth (in the sense that no non-smooth effects occur in the phase plot in Fig. 5c). Indeed, the two beads oscillate in synchronicity and in out-of-phase fashion, and at the precise time instant when they loose contact with the rigid walls they get into contact and start interacting with each other. On the other hand, when they detach from each other, they get into contact with the walls. Hence, at any instant of time a bead would either be interacting with the wall or with the other bead and the dynamics is smooth. Moreover, both beads cross their equilibrium positions at the same instant of time, so this mode conforms to the Rosenberg's definition of NNM [6]. It is worth mentioning that for higherdimensional systems the out-of-phase mode does not conform to the Rosenberg's definition of NNM [6]. In case of even number of beads, the pair of beads, which are positioned symmetric about the center of mass of the chain, would pass through their equilibrium points at the same instant of time.

Due to the fact that the out-of-phase NNM corresponds to synchronous and symmetric oscillations of the two beads, it can be explicitly analyzed. To this end, the oscillation is divided into two phases, during which the strongly nonlinear equations of motion decouple and can be explicitly solved. In the first phase of the oscillation, the total energy $h$ of the granular system is partitioned equally between the two beads in the form of elastic potential energy due to their interactions with the rigid walls. Hence, in this phase the two beads remain in contact with the walls and are detached from each other. Consequently, the mutual interaction terms in the equations of motion vanish and the equations of motion decouple completely:

$X_{1}^{\prime \prime}=\left(-X_{1}\right)_{+}^{3 / 2}$

$X_{2}^{\prime \prime}=-\left(X_{2}\right)_{+}^{3 / 2}$

subject to the initial conditions $X_{1}(0)=-(5 h / 4)^{2 / 5}$, $X_{1}^{\prime}(0)=0, X_{2}(0)=(5 h / 4)^{2 / 5}$, and $X_{2}^{\prime}(0)=0$. Denoting $X_{1}(\tau)=-X_{2}(\tau) \equiv X(\tau)$, the solution of (4) is computed in explicit form as

$$
\begin{aligned}
\tau= & h^{-1 / 10}(5 / 4)^{2 / 5}{ }_{2} F_{1}\left(\frac{1}{2}, \frac{2}{5} ; \frac{7}{5} ; 1\right. \\
& -\frac{X_{2}}{\sqrt{h}}{ }_{2} F_{1}\left(\frac{1}{2}, \frac{2}{5} ; \frac{7}{5} ; \frac{4 X_{2}^{5 / 2}}{5 h}\right.
\end{aligned}
$$



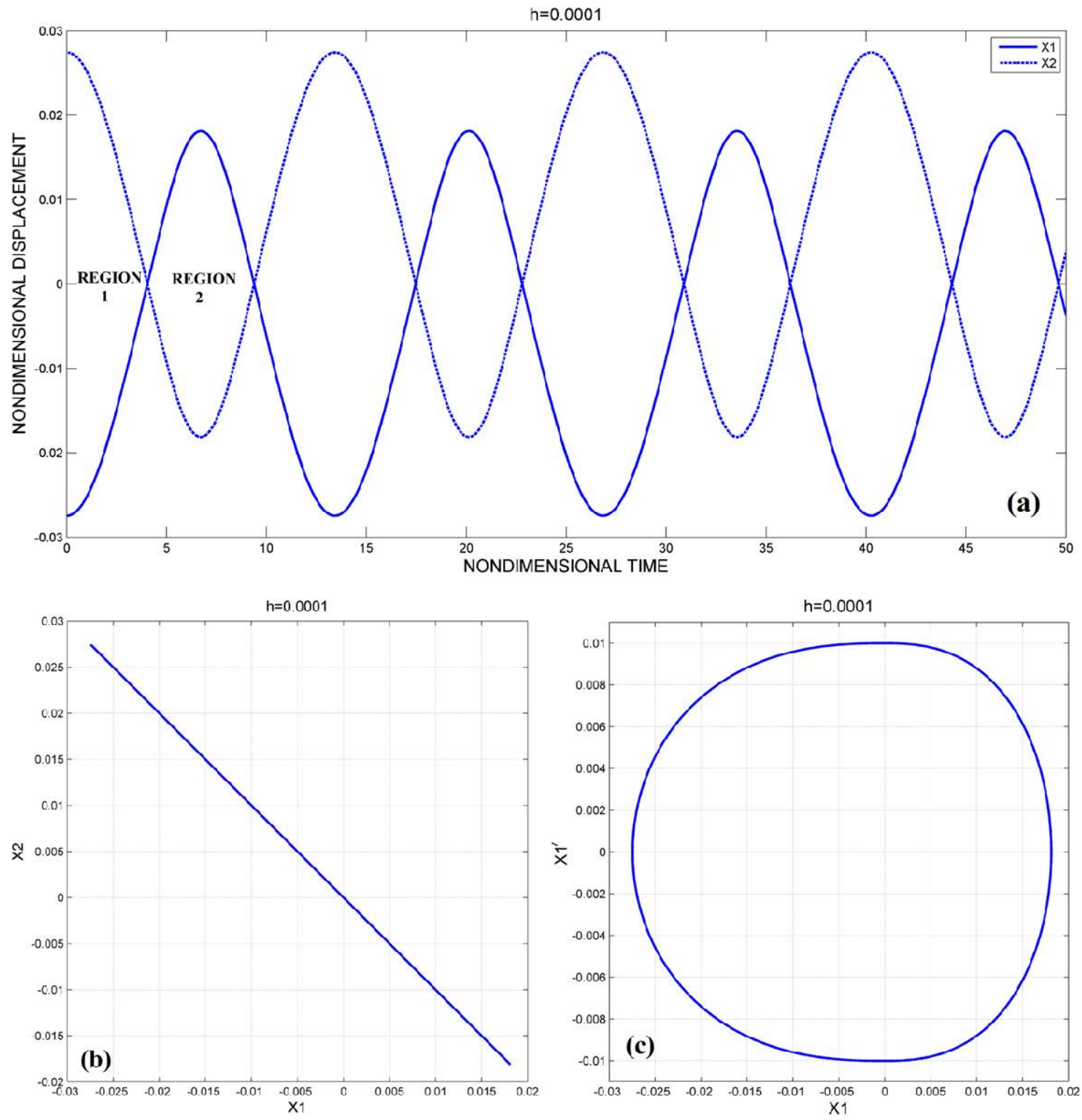

Fig. 5 The out-of-phase NNM for the two-bead granular system and $h=0.0001$ : (a) time series, (b) modal curve in the configuration plane, (c) depiction in a projection of the phase plane

where ${ }_{2} F_{1}(\bullet, \bullet ; \bullet ; \bullet)$ is a hypergeometric function defined as in [15],

$$
\begin{aligned}
{ }_{2} F_{1}(a, b ; c ; z)= & \frac{\Gamma(c)}{\Gamma(a) \Gamma(b)} \\
& \times{ }_{n=0}^{\infty}\left(\frac{\Gamma(a+n) \Gamma(b+n)}{\Gamma(c+n)}\right)\left(\frac{z^{n}}{n !}\right. \\
{ }_{2} F_{1}(a, b ; c ; 1)= & \frac{\Gamma(c) \Gamma(c-a-b)}{\Gamma(c-a) \Gamma(c-b)}
\end{aligned}
$$

and $\Gamma(\bullet)$ is the Gamma function [15]. This solution is valid only until the two beads reach their respective equilibrium points and the first phase of the oscillation is completed.

At that time instant the second phase of the oscillation starts. As the beads pass through their equilibrium positions, they lose their contact with the rigid walls and engage in mutual interaction. Again, the equations of motion can be combined into one and solved 
in closed form. To show this, we consider again the equations of motion

$X_{1}^{\prime \prime}=-\left(X_{1}-X_{2}\right)_{+}^{3 / 2}$

$X_{2}^{\prime \prime}=\left(X_{1}-X_{2}\right)_{+}^{3 / 2}$

and introduce the relative displacement variable $\delta=$ $X_{1}-X_{2}$. Then, the equations of motion can be combined into the following single equation:

$\delta^{\prime \prime}=-2(\delta)_{+}^{3 / 2}$

which can be solved explicitly by quadratures. We note at this point that at the end of the first phase of the oscillation the total energy of the system, $h$, is in the form of kinetic energy, equally distributed between the two beads. Hence, at the beginning of the second phase of the oscillation the beads have opposite velocities, which provides us with the necessary initial conditions, $\delta(0)=0, \delta^{\prime}(0)=2 \sqrt{h}$. Hence, the following analytical solution for (7) is derived:

$\tau=\frac{\delta}{2 \sqrt{h}} 2 F_{1}\left(\frac{1}{2}, \frac{2}{5} ; \frac{7}{5} ; \frac{2 \delta^{5 / 2}}{5 h}\right.$

The time period of the out-of-phase NNM can be similarly evaluated as

$$
\begin{aligned}
T & =T(h) \\
& =h^{-1 / 10}{ }_{2} F_{1}\left(\frac{1}{2}, \frac{2}{5} ; \frac{7}{5} ; 1\right)\left[2 \left(\frac{5}{4}^{2 / 5}+\left(\frac{5}{2}{ }^{2 / 5}\right.\right.\right. \\
& \approx 5.341 h^{-0.1}
\end{aligned}
$$

which provides an explicit expression for the dependence of the frequency $\omega=2 \pi / T$ of the out-of-phase NNM on the energy $h$. This analytical solution will be further utilized in our later discussion of the dynamics of the granular system in the frequency-energy plane.

The in-phase NNM corresponds to asynchronous and non-smooth oscillations of the two beads and it does not lend itself to a similar explicit solution. In fact, as many as five distinct phases of the dynamics exist for the in-phase NNM, of which only two can be analyzed explicitly (the other three phases correspond to concurrent interactions of the beads with each other and the rigid walls). This prevents the decoupling of the nonlinear equations of motion and a closed-form solution.

Focusing now on the subharmonic orbits of the two-bead system, in Figs. 6a-6c we present three representative oscillations corresponding to $1: 3,2: 3$ and 3:5 rational relations between the frequencies of the oscillations of the first and second beads, respectively. All these orbits are stable but the domains of quasi-periodic ('regular') motions surrounding them are small (cf. Fig. 2). Moreover, due to the nonintegrability of the dynamics of this system there exists a countable infinity of stable subharmonic orbits satisfying general $m: n$ rational frequency relationships, albeit with increasingly smaller domains of realization as the mutually prime integers $m$ and $n$ increase.

The two NNMs and subharmonic orbits can be better represented in a frequency-energy plot (FEP). As discussed in [17], by depicting the dynamics of a system in a FEP it is possible to study the influence of these modes on the forced and damped dynamics; moreover, it would be possible to better relate the dynamics of the two-bead granular system to the dynamics of higher-dimensional granular systems that will be considered later. To depict the two NNMs in the FEP it is necessary to compute their continuations for varying energy and derive the cor to responding frequency-energy relationships. This can be performed immediately for the out-of-phase NNM by means of relation (9). Since no similar explicit relationship can be derived for the in-phase NNM, we formulated a numerical shooting method to study this NNM for higher energy ranges. The shooting method is applied by specifying initial displacements and zero initial velocities for the two beads, so that at $\tau=0$ the entire energy is stored in the second bead due to its elastic compression by the right rigid wall (cf. Fig. 7). It follows that at $\tau=0$ the first bead is neither in contact with the wall nor with the second bead. Hence, assuming that the total (conserved) energy of the system is equal to $h$, the initial conditions for the granular system are chosen as

$$
\begin{aligned}
& X_{2}(0)=(5 h / 2)^{2 / 5}, \quad X_{1}(0)=\alpha_{1}^{(2)}(5 h / 2)^{2 / 5} \\
& X_{2}^{\prime}(0)=0, \quad X_{1}^{\prime}(0)=0
\end{aligned}
$$

where $0<\alpha_{1}^{(2)}<1$ is a constant that determines the asymmetry in the initial conditions for the in-phase mode, and it is computed by the shooting method. This constant is computed as $\alpha_{1}^{(2)} \approx 0.5585$, and it is interesting to note that it remains constant for all energies. This is due to the homogeneous nature of the system according to the definition of Rosenberg [6]. To per- 

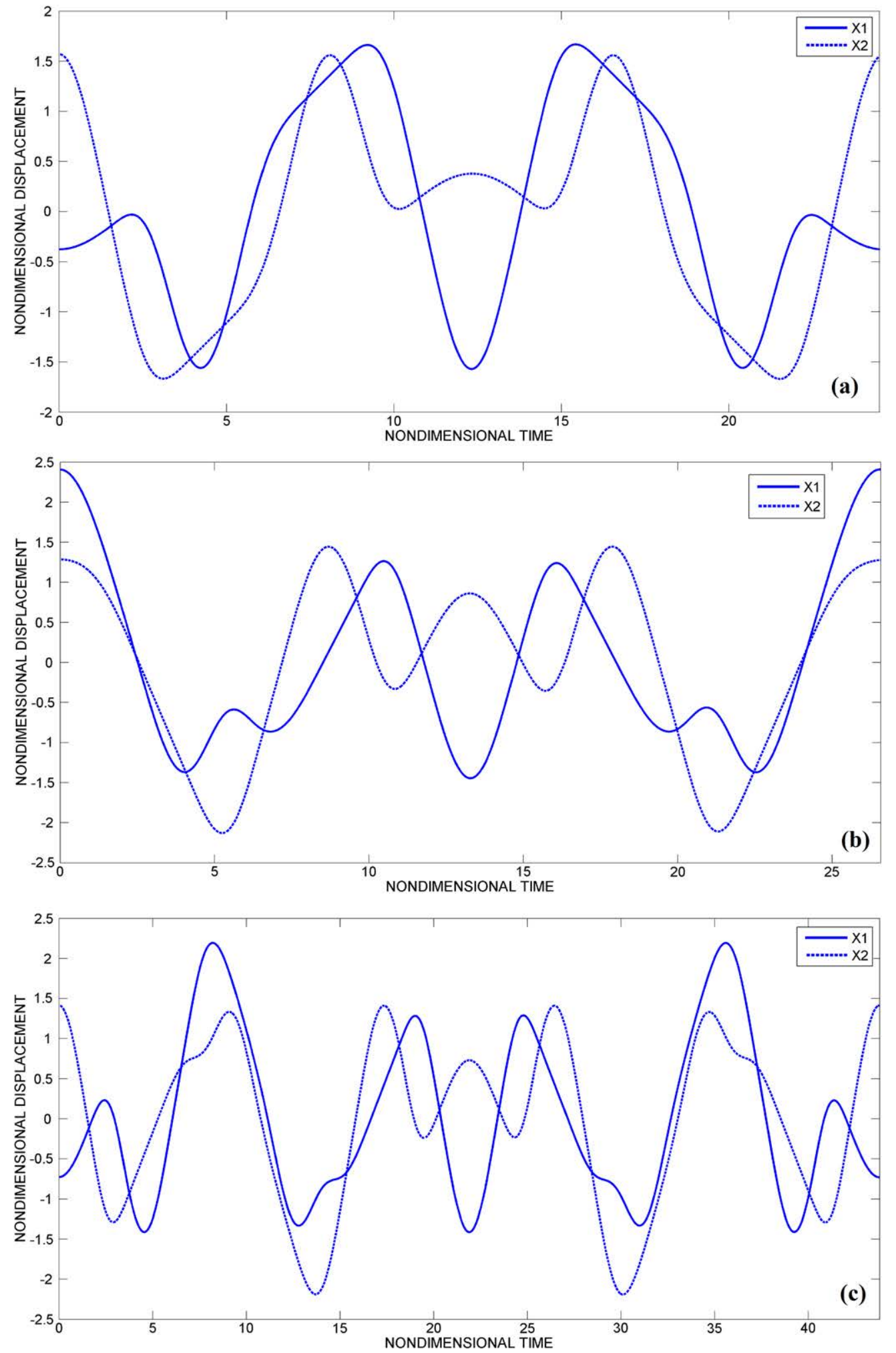

Fig. 6 Subharmonic orbits of the two-bead system and $h=0.0001:$ (a) 1:3, (b) 2:3, and (c) 3:5 ratios between bead frequencies 
form numerical continuation of the subharmonic orbits for higher energies we utilized the numerical algorithm developed by Peeters et al. as discussed in [17].

In Fig. 8 we present the different families of modes (NNMs and subharmonic orbits) of the two-bead system in the FEP. The frequency-energy curve of the out-of-phase NNM provides the upper limit of timeperiodic orbits for this system, so no periodic oscillations can be realized in the upper region of the FEP, which is labeled as 'prohibited' band. We note that the families of the two NNMs and subharmonic orbits are defined over the entire energy range and are represented by smooth curves that bifurcate from the origin of the FEP. This is in contrast to dynamical systems with essential but smooth stiffness nonlinearities, where subharmonic orbits appear as 'tongues' over finite energy intervals [17]. It will be of interest to study how the topological structure of the FEP changes as we increase the number of beads, and in particular, how the 'prohibited' band changes as the number of beads tends to infinity and the granular chain becomes

Fig. 7 Initial conditions for applying the shooting method for the in-phase NNM (two-bead system)

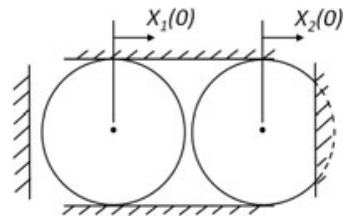

of infinite extent. These questions are discussed in the following sections.

\section{Three-bead granular system}

Considering the three-bead granular system and assuming identical elastic and geometric properties for the beads, the governing normalized equations of motion are given by

$$
\begin{aligned}
& X_{1}^{\prime \prime}=\left(-X_{1}\right)_{+}^{3 / 2}-\left(X_{1}-X_{2}\right)_{+}^{3 / 2} \\
& X_{2}^{\prime \prime}=-\left(X_{2}-X_{3}\right)_{+}^{3 / 2}+\left(X_{1}-X_{2}\right)_{+}^{3 / 2} \\
& X_{3}^{\prime \prime}=-\left(X_{3}\right)_{+}^{3 / 2}+\left(X_{2}-X_{3}\right)_{+}^{3 / 2}
\end{aligned}
$$

where the previous normalizations hold. In this case it is not feasible to compute Poincaré maps for studying the global dynamics since the dimensionality of the system is relatively high (it has a six-dimensional phase space). Nevertheless, it is possible to numerically compute the NNMs and subharmonic orbits of this system using the computational techniques discussed in the previous section.

Since the in-phase NNM (labeled as 'NNM 1') plays an important role in the dynamics of the granular system, we will examine this mode in detail. To

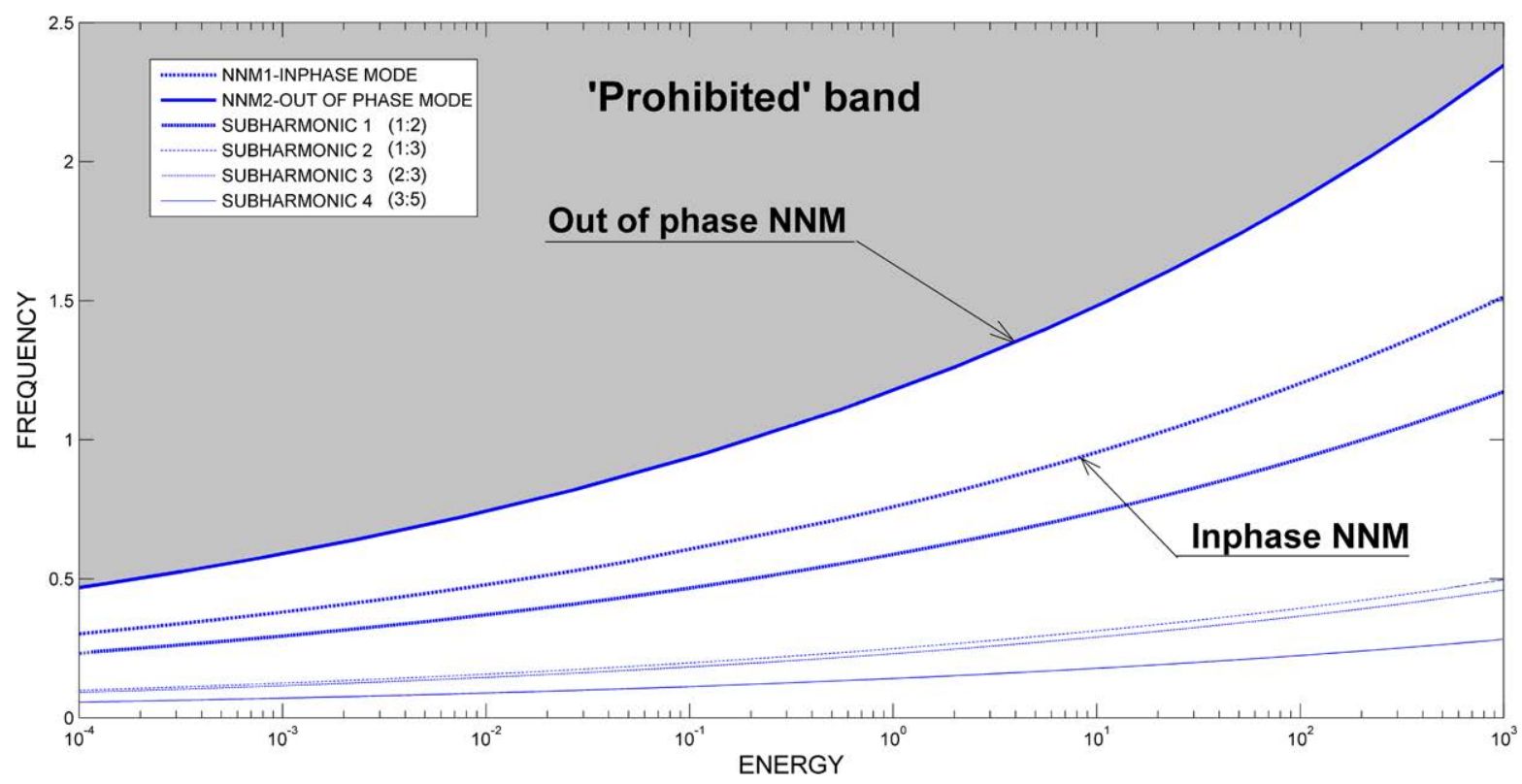

Fig. 8 Representation of the modes of the two-bead system in the frequency-energy plot (FEP) 
compute this NNM we employ the shooting method by assuming zero initial velocities and initial displacements in the form

$$
\begin{aligned}
& X_{3}(0)=(5 h / 2)^{2 / 5} \\
& X_{2}(0)=\alpha_{2}^{(3)}(5 h / 2)^{2 / 5} \\
& X_{1}(0)=\alpha_{1}^{(3)}(5 h / 2)^{2 / 5}
\end{aligned}
$$

where $h$ is the (conserved) energy of the system, and, as previously, the coefficients $\alpha_{1,2}^{(3)}$ characterize the asymmetry in the initial deformation of the in-phase NNM. These coefficients are computed as $0<\alpha_{1}^{(3)} \approx$ $0.374<\alpha_{2}^{(3)} \approx 0.744<1$ for all values of the energy $h$. In Figs. 9a-9c we depict the in-phase NNM; in similarity to the two-bead system, we infer that there are domains where beads become motionless and off-
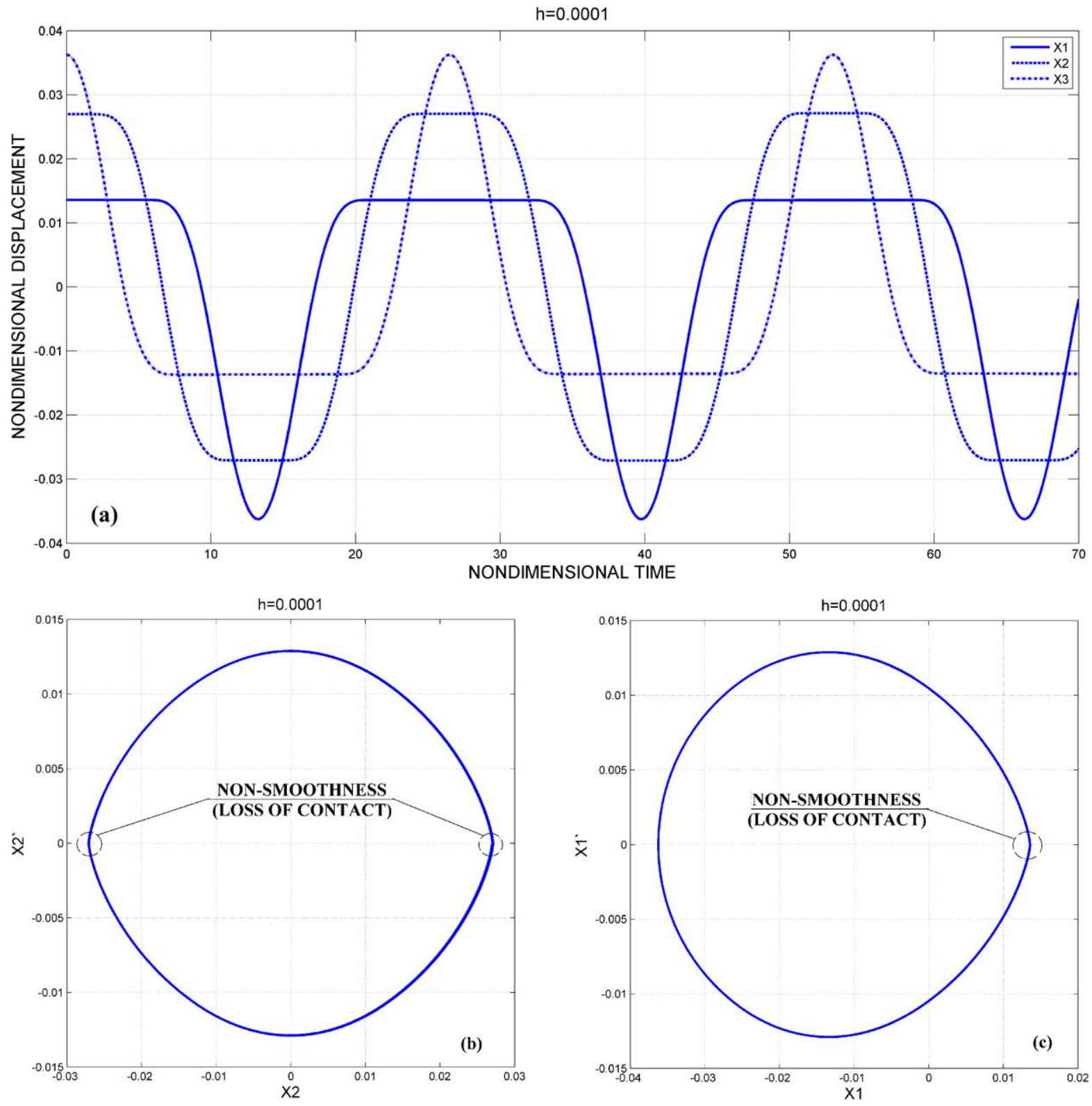

Fig. 9 The in-phase NNM (pseudo-traveling wave) for the three-bead granular system and $h=0.0001$ : (a) time series, (b, c) depictions in projections of the phase plane 


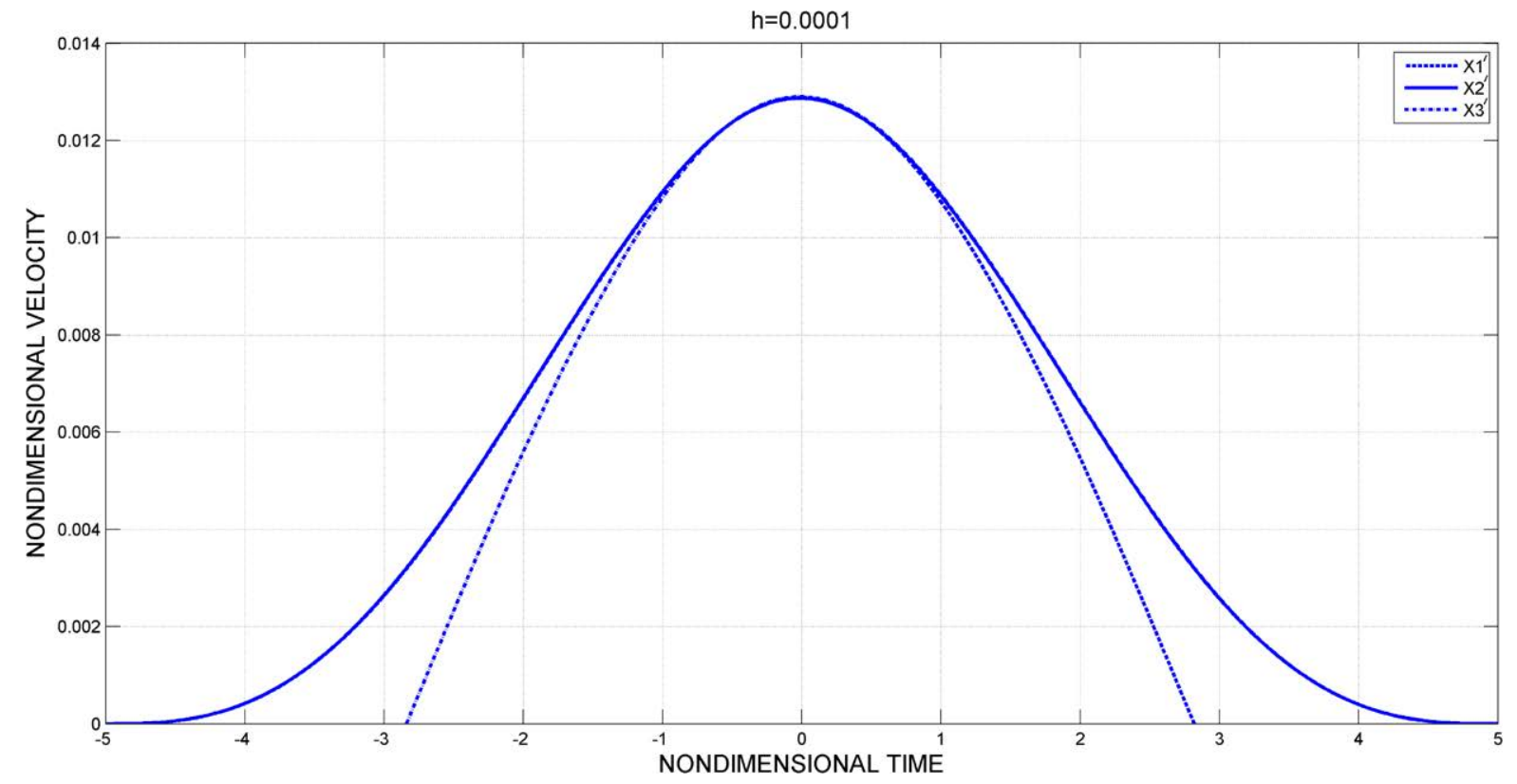

Fig. 10 Velocity profiles for the in-phase NNM (pseudo-traveling wave) of the three-bead granular system and $h=0.0001$

set from their zero equilibrium positions. In addition, non-smooth effects in the dynamics are clearly noted, and a high non-synchronicity between bead oscillations is deduced. The most important (and unique) features of this mode are the patterns of separation and loss of contact between beads and between the end beads and the rigid walls (which lead to the nonsmooth effects depicted in Figs. 9b, 9c). Indeed, the initial conditions required for realization of the inphase NNM are such that except for one of the beads, all other beads are detached from each other and with the walls. It is this feature that prevents the study of this mode using continuum approximation approaches in higher-dimensional systems with increased number of beads. Hence, the in-phase NNM is the mode most affected and influenced by the discrete nature of the granular system.

An even more peculiar feature of the in-phase NNM is that it resembles a traveling wave propagating back and forth in the granular chain. This becomes more apparent in higher-dimensional granular media (see the next section), but a first hint is provided by studying the velocity profiles in Fig. 10 for the three-bead system. In that figure we present a superposition of the velocity profiles of the central and end beads and show them on the same timescale. We note that the velocity profiles are in the form of single-hump 'pulses'; half of the velocity profiles of the end beads match exactly that of the central bead, whereas the other half is strongly influenced by the interaction of each end bead with the wall. Moreover, there is a constant time delay between the transmission of velocity 'pulses' in neighboring beads, so the in-phase NNM resembles a traveling wave. This result will be generalized for higher-dimensional systems where it will be shown that the velocity profiles of all beads except for the two end beads are identical but for a constant time shift. Hence, although the in-phase NNM is in actuality a time-periodic standing wave, the motion of each bead is followed by an extended period where it settles to an offset stationary position until the bead executes a motion in the reverse direction after a time interval equal to the half period of the NNM. Since each bead (except for the end ones) executes an identical motion but for a constant time shift, the in-phase NNM appears indeed as a traveling wave. Based on these observations we will refer from here on to the in-phase NNM as a pseudo-wave.

The second NNM (labeled as 'NNM 2') of the three-bead system corresponds to out-of-phase motions between neighboring beads and is presented in Fig. 11. Due to non-synchronicity of the oscillations of the three beads, this mode is again in non-conformance to the definition of NNM given by Rosenberg [6], 


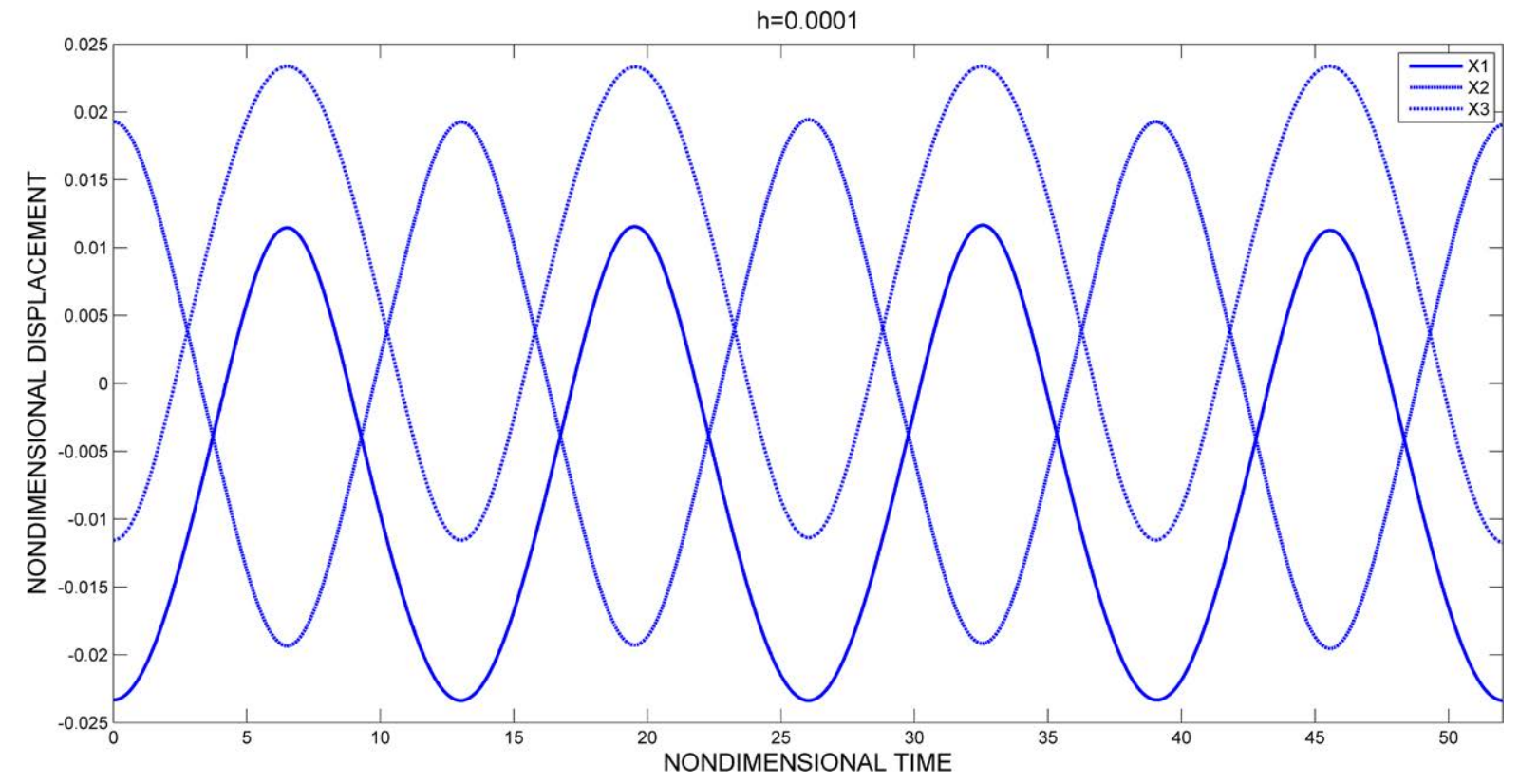

Fig. 11 The out-of-phase NNM for the three-bead granular system and $h=0.0001$

but in similarity to the two-bead case it represents the highest frequency NNM of the three-bead system, and as such, it forms the upper bound of the domain of periodic motions in the frequency-energy plane.

The third mode (labeled as 'NNM 3') of the threebead system corresponds to stationarity of the central bead (bead 2) for all times. As shown in Fig. 12, the dynamics of the system can be partitioned into two phases. In the first phase, the end beads share equally the energy of the system, which at $\tau=0$ is purely elastic. At the end of the first phase, the energy is completely transformed to kinetic and the two end beads have opposite velocities. Since the central bead is stationary, for this NNM it acts as a virtual wall. Hence, the dynamics of the system in the second phase is quite similar to the first one, and the equations of motion decouple throughout enabling us to solve for the bead responses in closed form. Omitting the details of the analysis, we may express the period of this NNM as

$$
\begin{aligned}
T & =T(h)=4 h^{-1 / 10}\left(\frac { 5 } { 4 } { } _ { 2 } ^ { 2 / 5 } F _ { 1 } \left(\frac{1}{2}, \frac{2}{5} ; \frac{7}{5} ; 1\right.\right. \\
& \approx 6.436 h^{-0.1}
\end{aligned}
$$

where $h$ denotes the total energy.
The last type of NNM (labeled NNM 4) supported by the three-bead system is localized, with one of the end beads interacting with the wall and oscillating with an amplitude that is much larger (about twice) than the corresponding amplitudes of the other two beads (cf. Fig. 13). In addition, neighboring beads oscillate in an out-of-phase fashion. It is clear that due to the symmetry of the system this mode is degenerate as it may be realized in an alternative symmetric configuration where the motion is localized to the other end bead. It is interesting to note that this type of nonlinear localization occurs in the homogeneous granular system, and in complete absence of pre-compression. To the authors' knowledge, this is the first report of this type of (strongly) localized motion in a single bead of a finite homogeneous granular system. We make the remark at this point that nonlinear localization in granular homogeneous media has been reported in previous works (e.g., $[18,19])$ but only under sufficiently strong pre-compression and/or in the presence of disorder, so that no separation between beads would be possible; moreover, spatially periodic standing waves with recurring localization after a wavelength have been reported in infinite granular chains with no pre-compression [16]. We were not able to detect an analogous localized NNM where the localiza- 


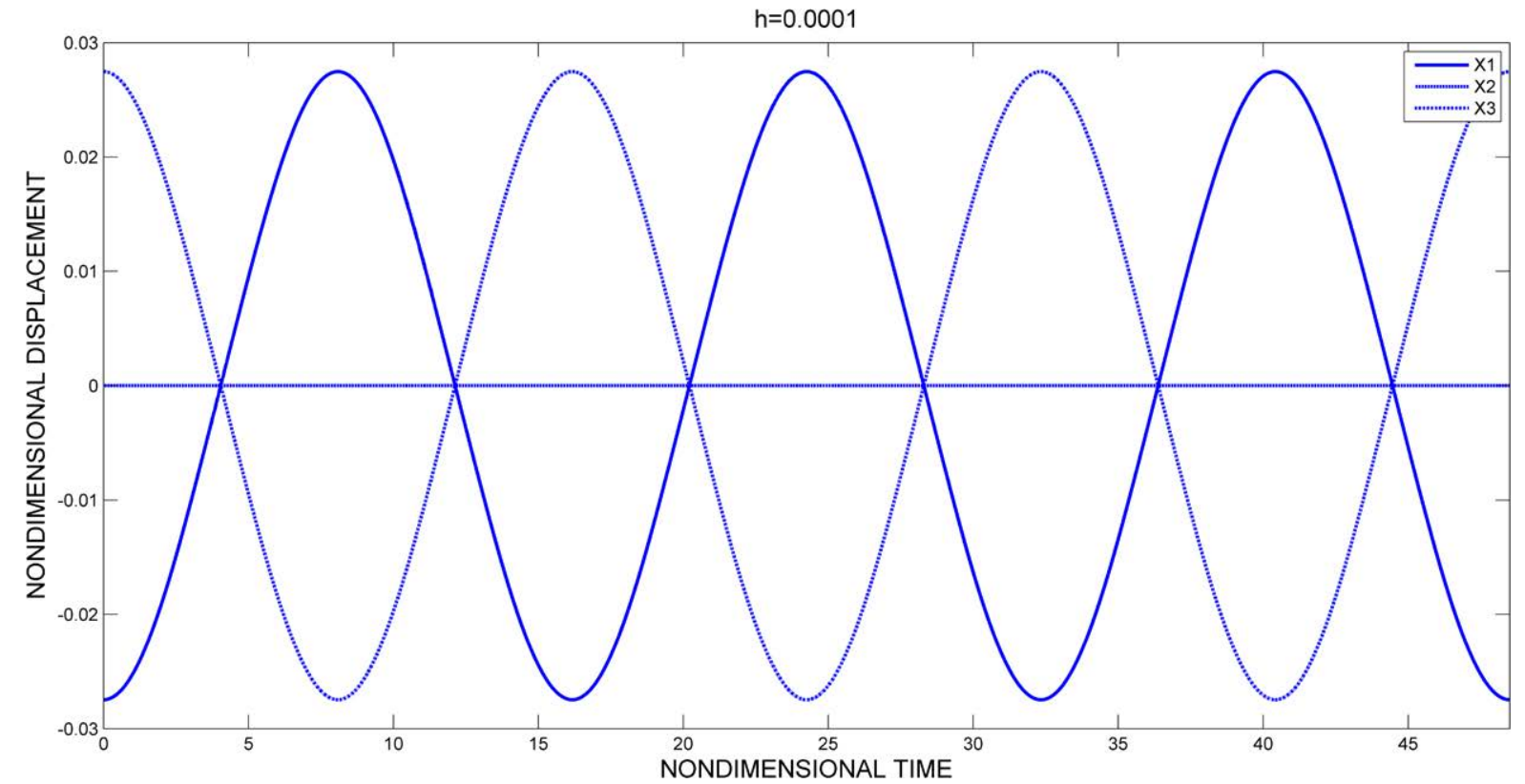

Fig. 12 The NNM with stationarity of the central bead for the three-bead granular system and $h=0.0001$

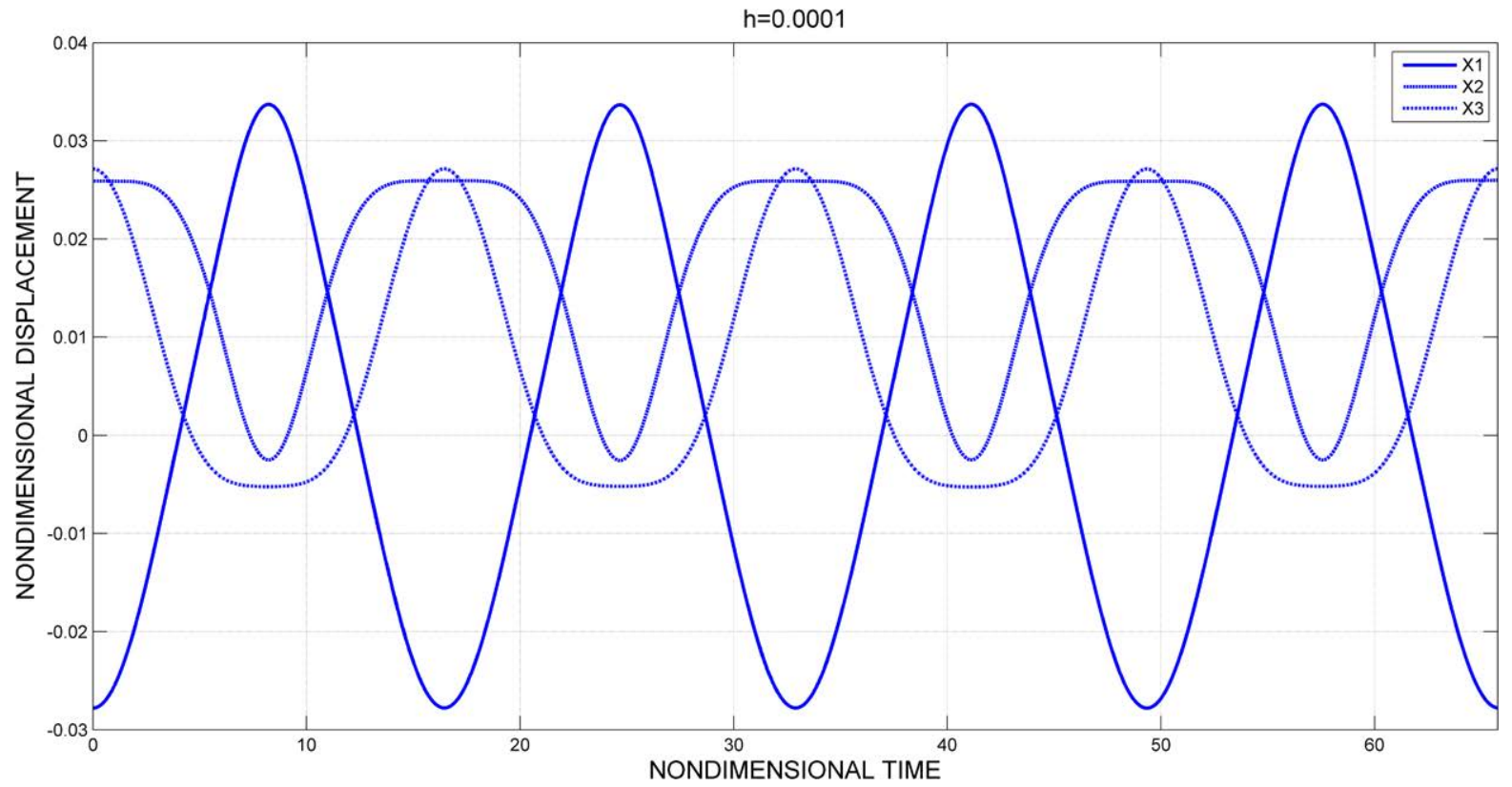

Fig. 13 The localized NNM for the three-bead granular system and $h=0.0001$

tion takes place in the central bead of the three-bead system under consideration.

In addition to the four NNMs, the system supports a countable infinity of subharmonic orbits, in similarity to the two-bead system. The FEPs of the NNMs and subharmonic orbits of the three-bead system are presented in Fig. 14. From the discussion above, the number of NNMs in this system exceeds its degrees of freedom, but this is possible in essentially nonlinear discrete oscillators [7]. The upper boundary separat- 


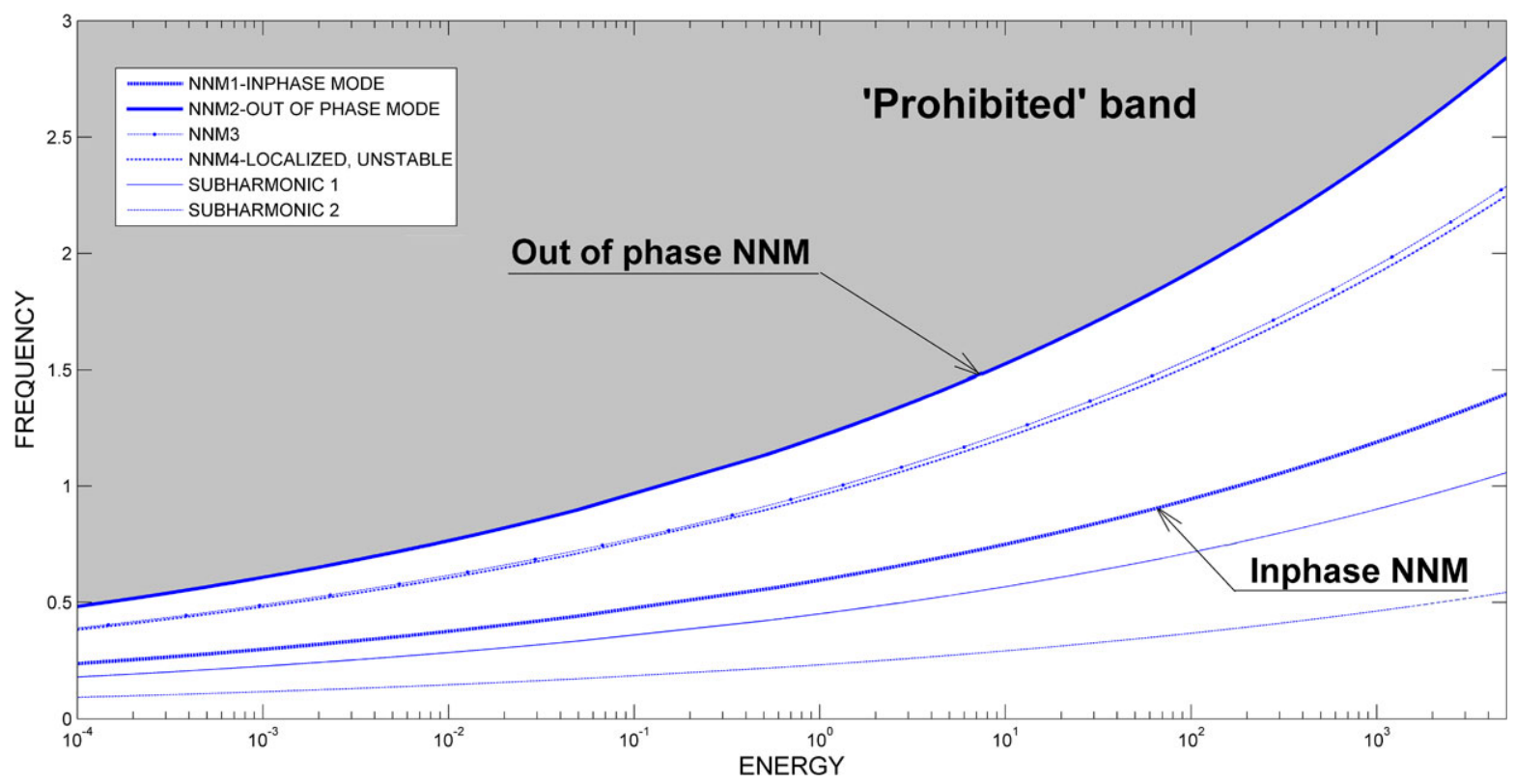

Fig. 14 Representation of different modes of the three-bead system in the frequency-energy plot (FEP)

ing the region of time-periodic orbits from the 'prohibited' band, again, is formed by the out-of-phase NNM 2, whereas the lowest frequency mode is the in-phase NNM 1, which as discussed previously is a pseudo-wave. Moreover, compared to the corresponding results of the two-bead system (cf. Fig. 8) we note that with increasing number of beads the upper boundary (corresponding to the out-of-phase NNM) moves toward higher frequencies, whereas the curve corresponding to the in-phase (pseudo-wave) NNM moves toward lower frequencies. This trend will be confirmed in the next section where higher-dimensional granular systems are considered. In the FEP of Fig. 14 we depict also the frequency-energy curves of two subharmonic orbits, which occur within the complement of the 'prohibited' band. No time-periodic orbits (NNMs or subharmonic orbits) can occur in the 'prohibited' band, similarly to the case of the two-bead system.

\section{Higher-dimensional granular systems}

We now extend our analysis to homogeneous granular chains of higher dimensionality. Our main aim is to study the changes in the structure of the FEP as the number of beads increases, and, in particular, to identify regions in the frequency-energy plane where spatial transmission of disturbances (i.e., energy) is facilitated or prohibited by the intrinsic dynamics of the granular medium. This information is of practical significance when such media are designed as passive mitigators of shocks or other types of unwanted disturbances. Since the out-of-phase and inphase NNMs represent the highest and lowest frequency NNMs of the granular medium, respectively, all other NNMs (localized or non-localized) are realized in the frequency-energy band defined by these 'bounding' NNMs, irrespective of the dimensionality of the granular medium. Hence, in what follows we will only focus on these two NNMs and investigate how the topology of the band of realization of NNMs (and its complementary high-frequency 'prohibited' band) changes with increasing number of beads in the granular chain.

In Fig. 15 we depict the in-phase and out-of-phase NNMs in the FEP for systems composed of two to seven beads. We note that as the number of beads increases the out-of-phase NNM makes a transition toward higher frequencies and quickly converges (accumulates) to a definite upper bounding curve, whereas the in-phase NNM makes a similar transition toward lower frequencies. No such quick convergence is noted for the in-phase NNM, but rather, as the number of beads tends to infinity, the in-phase NNM tends toward 


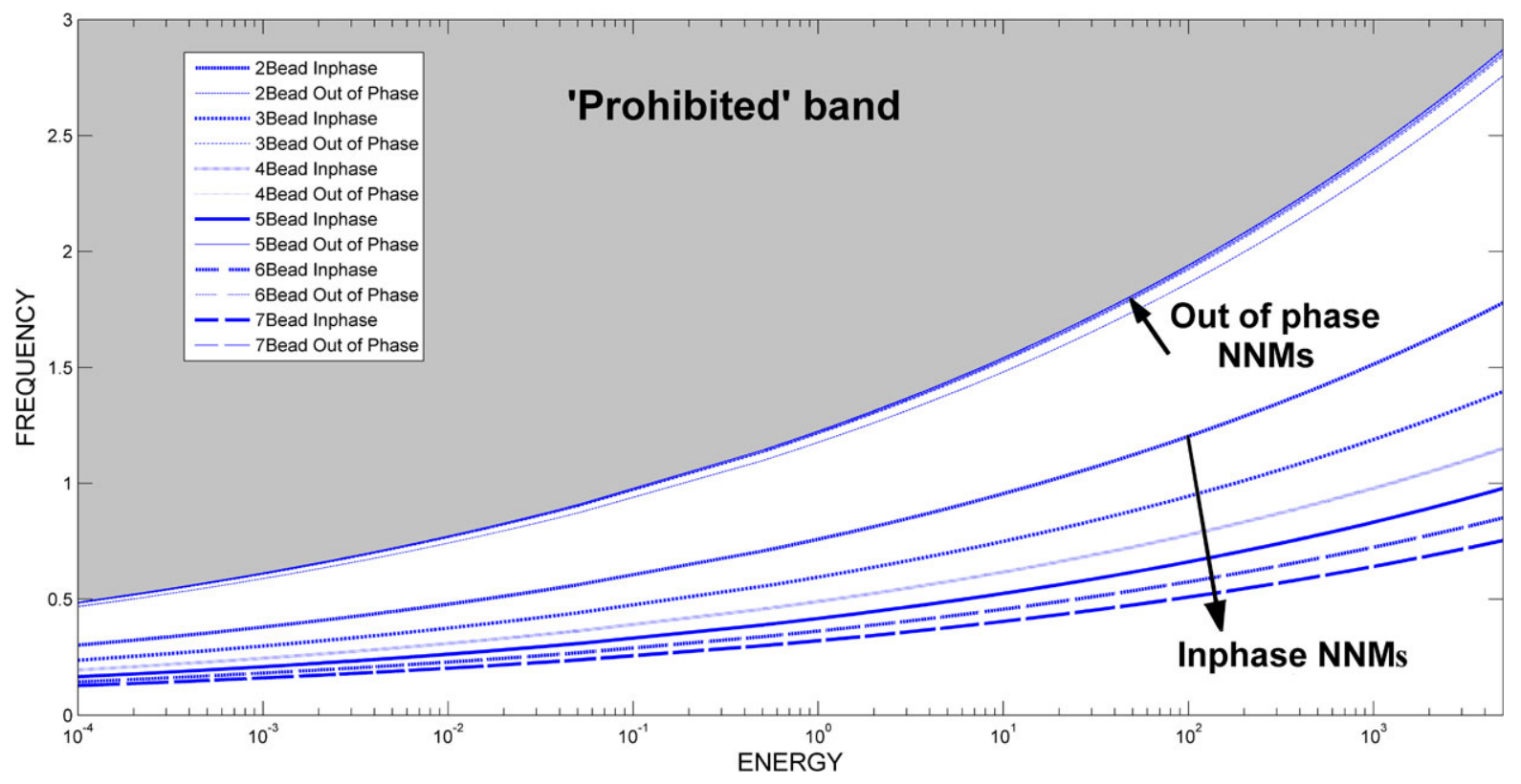

Fig. 15 Representation of the in-phase and out-of-phase NNMs in the frequency-energy plot (FEP) for granular systems from two to seven beads; in the limit of infinite number of beads, the FEP is partitioned into a propagation and an attenuation band

the zero-frequency axis. This raises an interesting question concerning the dynamics of the infinite granular system as the in-phase NNM approaches the zerofrequency limit. Namely, as discussed in the previous section, due to bead separation the in-phase NNM resembles a traveling wave corresponding to a singlehump velocity disturbance propagating back and forth through the granular system (hence the previous characterization of this NNM as a pseudo-wave). Moreover, the 'silent' period for each bead (corresponding to the time period where the bead remains motionless at an offset position) progressively increases with increasing dimensionality of the system. As discussed previously, in actuality the in-phase NNM is a stable standing wave, but for any specific bead the recurrence of the disturbance happens after a 'silent' period equal to half the period of the in-phase NNM; in turn, this period increases with increasing number of beads, as is the corresponding 'silent' period of the in-phase NNM. Following this argument one step further, one might deduce that in the limit of infinite number of beads the period of the NNM tends to infinity (and its frequency tends to zero as it approaches the energy axis in the FEP), so the in-phase mode should degenerate to a true traveling wave, similar (or identical?) to the solitary wave studied by Nesterenko [9].
In Fig. 16 we compare the velocity profile of the solitary wave studied by Nesterenko to the singlehump velocity profiles of the in-phase NNMs of fixedfixed three- to seven-bead granular systems. It is clear that the velocity profiles of the in-phase NNMs do not converge to the solitary wave studied by Nesterenko. Clearly, the in-phase NNM converges to a different limit as the dimensionality of the system tends to infinity, which raises an obvious question concerning the type of dynamics that this limit represents. The answer can be found by considering more closely the dynamics of the granular chain when it oscillates on the inphase NNM. As discussed previously, for an $n$-bead granular system the initial conditions necessary for exciting the in-phase NNM can be expressed in terms of non-zero initial normalized displacements and zero initial velocities as follows:

$$
\begin{aligned}
X_{1}(0) & =\alpha_{1}^{(n)}(5 h / 2)^{2 / 5}<X_{2}(0) \\
& =\alpha_{2}^{(n)}(5 h / 2)^{2 / 5}<\cdots<X_{n-1}(0) \\
& =\alpha_{n-1}^{(n)}(5 h / 2)^{2 / 5}<X_{n}(0) \\
& =(5 h / 2)^{2 / 5}
\end{aligned}
$$

where $h$ is the (conserved) energy of the system. This indicates that in order to realize the in-phase NNM, 


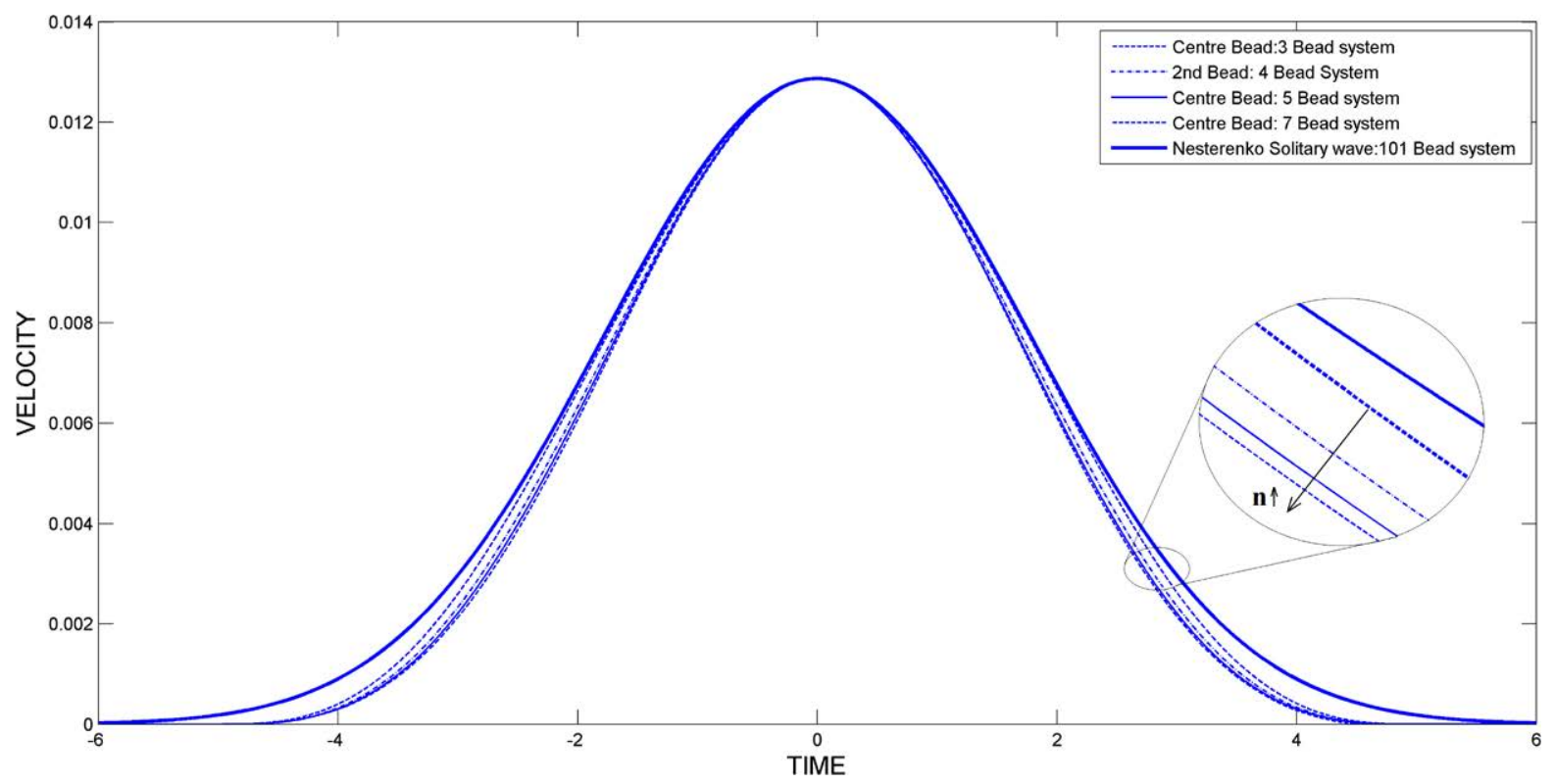

Fig. 16 Comparison of the velocity profiles of the in-phase NNMs of fixed-fixed $n$-bead granular systems to the velocity profile of the solitary wave studied by Nesterenko [9]

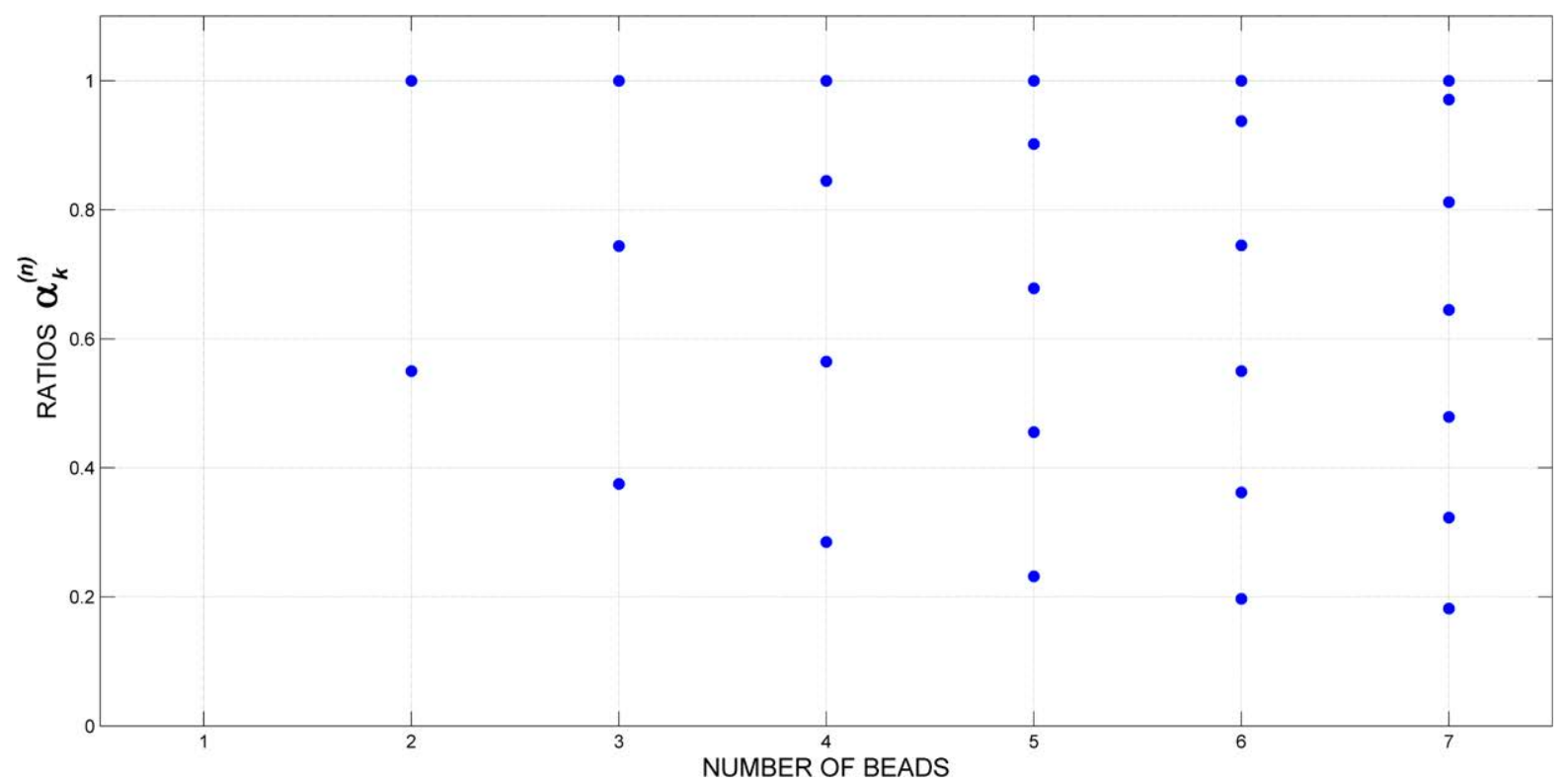

Fig. 17 Coefficients $\alpha_{k}^{(n)}, k=1, \ldots, n-1$, for systems with $n=2, \ldots, 7$ for the in-phase NNMs of the fixed-fixed granular system, with $\alpha_{n}^{(n)}=1$

it is necessary to compress the $n$th bead at the right end of the granular system, and then allow for gaps (clearances) between the rest of the beads, which monotonically decrease as the left end of the system is reached. In Fig. 17 we depict the coefficients $\alpha_{k}^{(n)}$, $k=1, \ldots, n-1$, characterizing the asymmetry in the in-phase NNM deformation (in terms of the corresponding gaps) for systems with $n=2, \ldots, 7$ beads, and note that as $n$ increases the differences between these coefficients decrease. Moreover, the numerical 

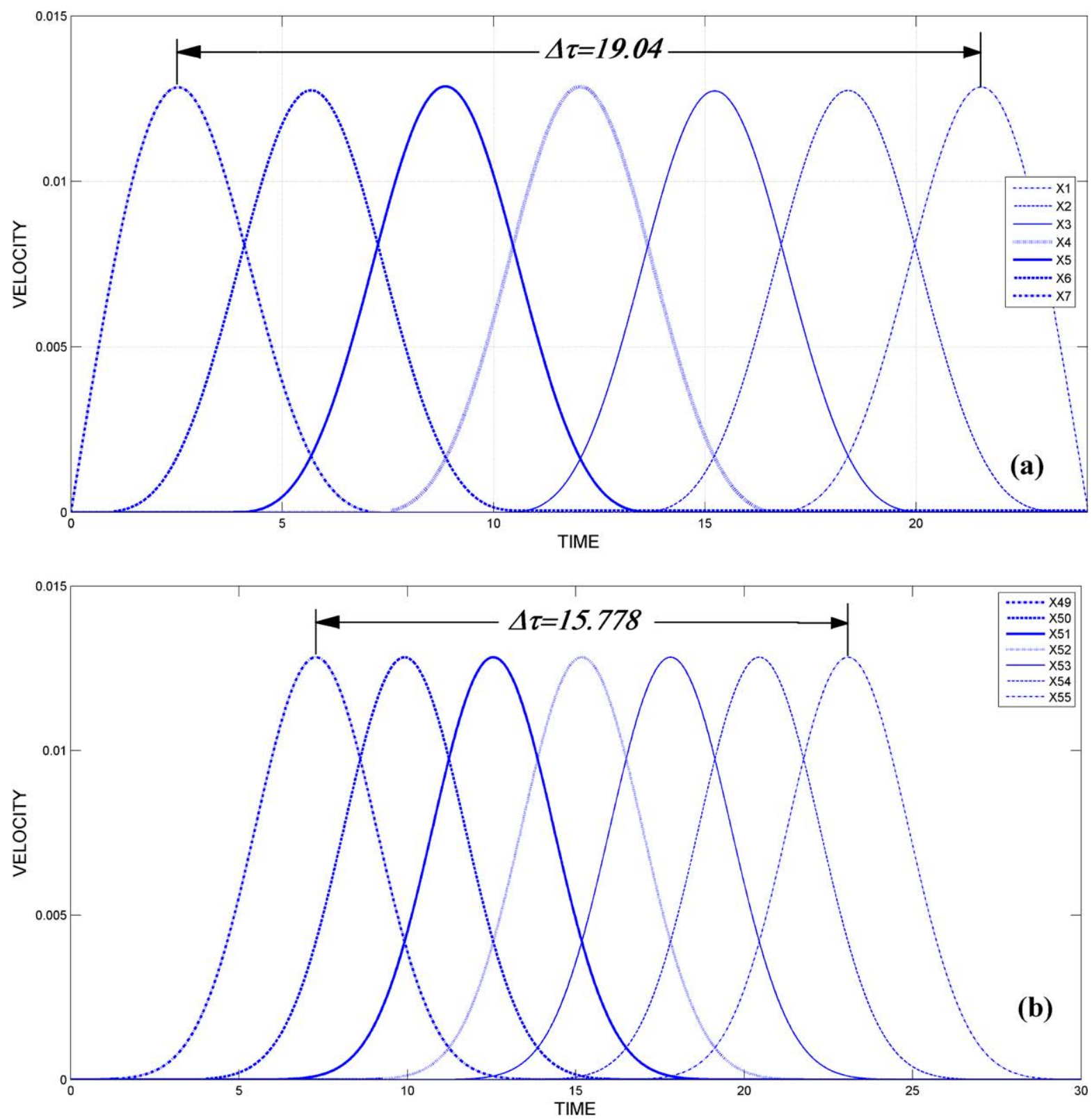

Fig. 18 Velocity profiles of seven adjacent beads: (a) in-phase NNM, (b) the solitary wave [9]; time delays in terms of normalized time $\tau$

results indicate that in the limit $n \rightarrow \infty$ these gaps should tend to zero, which indicates that the in-phase NNM cannot be realized in the limit of infinite granular chain. We conclude that the infinite limit represents a singularity for the family of in-phase NNMs, which makes physical sense since otherwise this family of NNMs (which are non-synchronous standing waves or pseudo-waves) would degenerate to an actual trav- eling wave, which would be a clear inconsistency in terms of the dynamics. We make the remark at this point that even though the realization of the in-phase NNM is hindered as the dimensionality of the granular chain increases, it can significantly influence the dynamics of lower-dimensional granular media.

To demonstrate more clearly the pseudo-wave character of the in-phase NNM, in Fig. 18 we present the 
Table 1 Normalized time delays for velocity pulse propagation

\begin{tabular}{lll}
\hline$n$ & $\begin{array}{l}\text { 100-Bead chain } \\
\text { (Solitary wave [9]) }\end{array}$ & $\begin{array}{l}n \text {-Bead system } \\
\text { (In-phase NNM) }\end{array}$ \\
\hline 3 & 5.248 & 7.67 \\
4 & 7.858 & 10.62 \\
5 & 10.528 & 13.45 \\
6 & 13.168 & 16.25 \\
7 & 15.778 & 19.04 \\
\hline
\end{tabular}

velocity profile over half the period of the mode for each of the beads of the seven-bead granular system with fixed-fixed boundary conditions, and compare it to the corresponding velocity profiles for seven beads for solitary wave propagation [9]. Since theoretically the solitary wave can be realized only in the infinite granular chain, the results in Fig. 18 were computed for a chain with 100 beads and free-free boundary conditions; the magnitude of the impulse provided to the first bead was selected so that the velocity amplitude of the resulting solitary wave matches the velocity amplitude of the in-phase NNM of the seven-bead fixedfixed granular system.

We note that, whereas the velocity profiles of all beads match exactly for the case of the solitary wave (cf. Fig. 18b), the same holds only for the velocity profiles of the five central beads for the case of the inphase NNM (cf. Fig. 18a). For the end beads of the fixed-fixed granular system, only half of their velocity profiles match those of the central beads, whereas the other half are strongly influenced by the interaction of these beads with the rigid walls. Moreover, comparing the time delays of arrival of the pulse from the first bead to the last, we note that the solitary wave possesses a higher speed compared to the pseudo-wave in the fixed-fixed granular system. We note that in Fig. 18a the pseudo-wave is depicted for only one half of the period of the in-phase NNM (for the other half of the period the pseudo-wave is 'reflected' by the right rigid wall and it 'propagates' in the opposite direction-i.e., backwards - through the medium). A tabulation of these time delays for systems with three to seven beads is presented in Table 1, from which we infer that the pseudo-wave (i.e., the inphase NNM) becomes slower compared to the solitary wave studied by Nesterenko with increasing number of beads.

\section{Intrinsic dynamics of the infinite granular chain: propagation and attenuation bands}

In the limit of infinite number of beads the frequencyenergy plane is partitioned into two regions, namely a propagation band $(\mathrm{PB})$ and an attenuation band $(\mathrm{AB})$. The existence of this type of bands in linear [2023] and nonlinear [19, 24] periodic media has been well documented in the literature. Typically, in PBs traveling waves exist, and these are the dynamical mechanisms for spatially transferring energy through the medium to the far field. In ABs near-field motions with exponentially decaying envelopes are realized that cannot propagate energy in the far field. The boundaries separating the $\mathrm{PBs}$ and $\mathrm{ABs}$ are spatially extended time-periodic standing waves, which can be regarded as NNMs of the infinite periodic media.

Similar types of orbits are realized inside the $\mathrm{PBs}$ and $\mathrm{ABs}$ of the infinite homogeneous granular medium with no pre-compression. The portioning of the FEP of the infinite granular chain in terms of PBs and $\mathrm{ABs}$ is realized by the limiting (accumulating) curves of the in-phase and out-of-phase NNMs as depicted in Fig. 15. As the number of beads tends to infinity, the in-phase NNM tends toward the energy axis (i.e., zero frequency), and the spacing between the frequency-energy curves of the NNMs and subharmonic orbits tends to zero (or alternately, the NNMs and subharmonic orbits become densely 'packed' in terms of frequency and energy inside the PB).

In the infinite limit, the dynamics of the granular medium possesses continuous families of traveling waves inside the PB. These motions were studied in [16], where it was shown that the infinite granular medium supports a countable infinity of families of stable traveling waves in the form of propagating multi-hump velocity pulses with arbitrary wavelengths; these families are parameterized by the 'silent' regions of zero velocity that separate successive maxima of the propagating velocity pulses. As these 'silent' regions increase, the corresponding wavelengths and periods of the traveling waves also increase and the frequencies of the waves decrease. In the limit of infinite 'silent' region, the wave ceases to be periodic and its frequency becomes zero; this asymptotic limit of single-hump solitary waves is the solitary wave studied by Nesterenko [9].

Different families of traveling waves were computed in [16] and their propagation properties were 
studied. It was found that these periodic waves are slower than the solitary wave studied by Nesterenko, with their speeds strongly depending on their amplitudes (energies). We wish to depict the frequencyenergy curves of these families of traveling periodic waves in the FEP of the infinite granular chain. Clearly, since each family of traveling waves corresponds to an infinite sequence of propagating velocity pulses separated by finite 'silent' regions (where no motion occurs), it carries an infinite amount of energy. In order to assign a finite energy measure to each family of traveling periodic waves, we computed the corresponding energy density, i.e., the energy carried by each wave family over a wavelength of the motion. Moreover, the corresponding frequency follows directly from the time-periodic character of the waves. As the 'silent' region of the traveling wave increases, so does its period. It follows that with increasing 'silent' region the frequency of the wave decreases, until in the limit of infinite 'silent' region (corresponding to the solitary wave studied by Nesterenko) the energy axis is reached and the frequency of the wave is zero. In addition, it is interesting to note that for all traveling waves with finite 'silent' regions there occur separations between beads, whereas in the limit of infinite 'silent' region there are no separations between beads; in that limit a continuum approximation can be utilized to study the solitary wave (as performed by Nesterenko in [9]).

In Fig. 19, we depict two of the families of traveling waves, which as discussed previously are distinguished by the regions of zero velocity (the 'silent' regions) between successive maxima of velocity pulses. One family corresponds to traveling waves with threebead periodicity, whereas the second to four-bead periodicity [16]. Indeed, we confirm that these families of waves are realized inside the PB of the infinite granular medium. In the limit of infinite 'silent' region, we obtain the single-hump solitary wave studied by Nesterenko, which lies on the energy axis of the FEP (as it corresponds to infinite period or zero frequency).

In addition to these families of traveling periodic waves, additional motions can occur inside the PB, including subharmonic motions, standing waves with recurring localization features (i.e., periodically spaced humps) and chaotic orbits. A family of such standing waves with three-bead periodicity [16] is depicted in the FEP in Fig. 19, with one of the beads oscillating in out-of-phase fashion and with higher amplitude com- pared to its neighboring beads. We conjecture that additional families of such standing waves occur inside the PB of the infinite granular medium, distinguished by the extent of bead-periodicity (spatial wavelength) of the standing wave, and type of localization characteristics. We end our discussion of the dynamics inside the PB by noting that no similar standing waves (with or without localization characteristics) can occur in PBs of perfectly ordered linear periodic media, although standing waves with spatially localized slopes can occur in ordered periodic media with smooth stiffness nonlinearities [26]. The full study of standing waves with localization features, however, is beyond the scope of this work.

We now consider the dynamics of the infinite granular system inside the attenuation band (AB) of the FEP in Fig. 19. No spatially-periodic standing or traveling waves can occur for frequency-energy ranges in that band, so no spatial transfer of energy in the granular medium is possible for motions inside the $\mathrm{AB}$. Rather, near-field motions occur inside the $\mathrm{AB}$, corresponding to spatially periodic oscillations of the beads about different positive offset positions with spatially decaying envelopes; overall, the motion of the granular medium is a standing wave with decaying envelope, with each bead performing a time-periodic oscillation about its own (positive offset) equilibrium position. Examples of this type of near-field standing waves are provided in the next section where the influence of the propagation and attenuation bands on the forced response of the granular medium to narrowband excitation is studied. We note that the response of the granular medium inside the $\mathrm{AB}$ resembles the responses of unforced linear periodic media [20-22], which possess similar decaying standing waves (and even decaying 'complex' waves when more than one coupling coordinates between individual substructures exist [25]) in well defined attenuation bands.

In the next and final section, we demonstrate the influence of the intrinsic dynamics of the unforced granular system on the forced dynamics of the same system forced by a narrowband excitation. We expect that the partitioning of the FEP in terms of propagation and attenuation bands will affect in a significant way the capacity of the granular medium to transmit or attenuate disturbances through it. This, in turn, has obvious implications on the capacity of the granular chain to act as passive mitigator of unwanted disturbances. 
Fig. 19 Traveling and standing waves inside the propagation band (PB $\square$ ) of the FEP of the infinite granular chain [16], the attenuation band (AB $\mathbf{\square})$ is also indicated:

(a) frequency-energy curves, (b) velocity profiles of traveling waves inside the PB, with three- and four-bead periodicity, (c) velocity profile of a standing wave inside the $\mathrm{PB}$, with three-bead periodicity
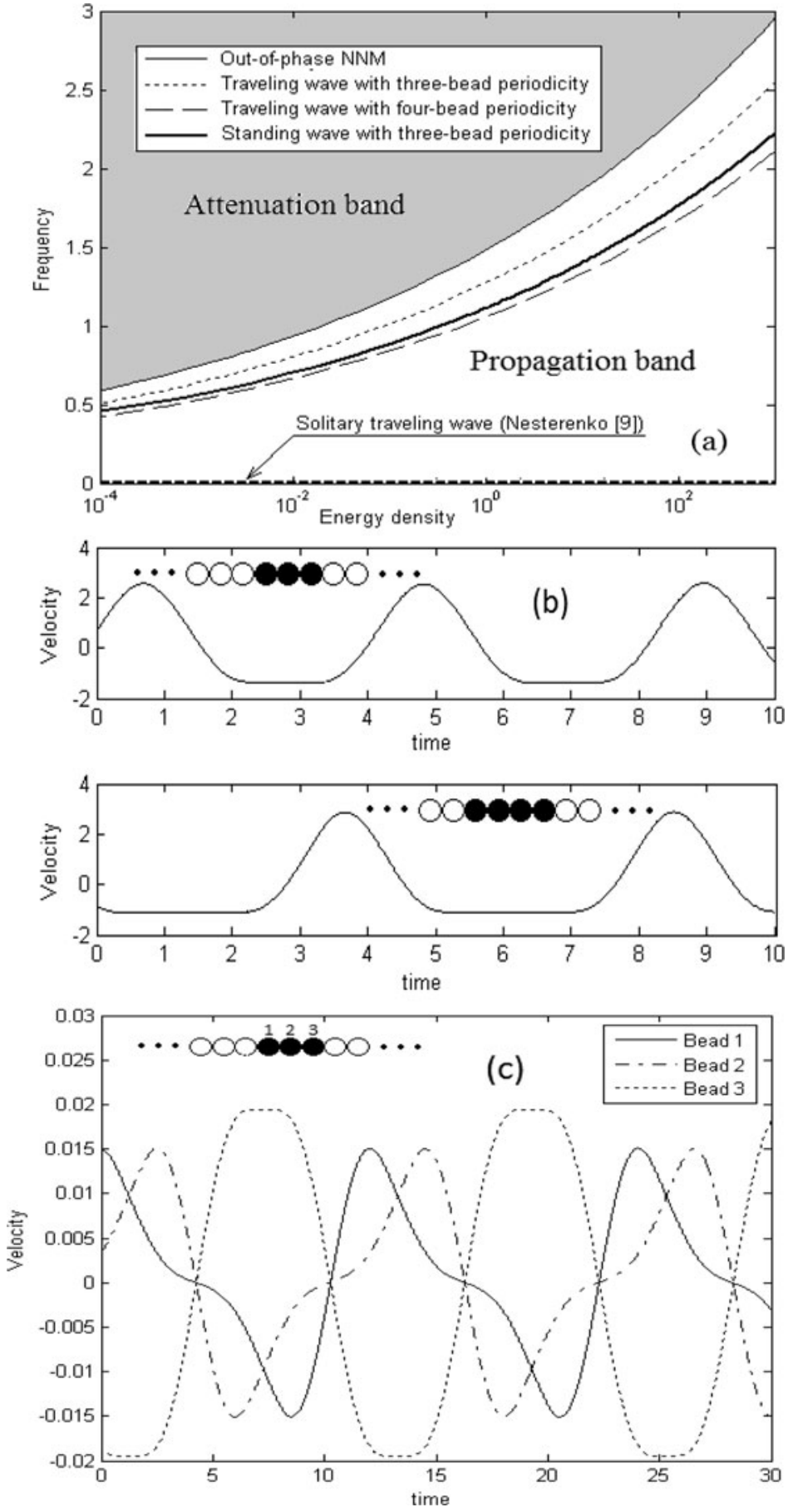

\section{Influence of the intrinsic dynamics on the forced responses of the granular medium}

In this final section, we show how the topology of the propagation and attenuation zones in the frequency- energy plot (FEP) affects the forced dynamics of the multi-dimensional granular chain. The influence of the intrinsic dynamics of the unforced chain on the forced dynamics is inferred from our previous observation that disturbances initiated inside the PB of the FEP can 
spatially transfer energy within the granular medium (through excitation of traveling waves), whereas motions initiated inside the $\mathrm{AB}$ are near-field solutions that cannot transfer energy through the medium. To numerically verify this theoretical prediction we excited a 50-bead granular chain by imposing a harmonic excitation on its left boundary in the form $y_{0}=$ $A \sin \omega \tau$, where $\omega$ is the cyclic frequency of the excitation in terms of the normalized time $\tau$. Although this is a finite-dimensional medium, we expect that, with the exception of certain end effects, its intrinsic dynamics will be close to the dynamics of the corresponding infinite chain. In order to eliminate high-frequency components in the response of the chain that result due to the excitation of low-amplitude chaotic motions (resulting from the strong non-integrability of the granular chain-see the Poincaré map in Fig. 2), we added weak viscous dissipative forces in the interactions between neighboring beads with viscous damping coefficients equal to $d=0.01$. As shown below, these weak dissipative terms successfully dampen out highfrequency chaotic contributions to the dynamics and help us reveal coherent features generated by the intrinsic dynamics in the forced responses.

In Fig. 20 we depict the forced response of the granular system for excitation parameters $A=1.5$ and $\omega=1$. This corresponds to dynamics that occur inside the PB of the infinite chain, and this is confirmed by the spatially extended dynamical response of the system. We note that the initial disturbance generated at the left boundary of the 50-bead chain is transmitted throughout the chain; in addition, the total energy in the system gradually builds up until it forms an oscillation about a high level. Moreover, from the snapshots of the chain deformation at different time instants presented in Fig. 20b, we infer that the initial disturbance travels from the left boundary through the chain and gets reflected from the right boundary. It follows that the forced dynamics of the chain is mainly caused by excitation of the spatially extended intrinsic motions, which, as discussed in the previous section, are densely 'packed' inside the PB of the FEP. Hence, we conclude that in this case, the intrinsic dynamics favor the transfer of disturbances through the chain.

A qualitatively different picture for the dynamics is inferred from the results in Fig. 21 that depict the forced response of the chain inside the $\mathrm{AB}$ of the FEP. In this case the excitation parameters are selected as for $A=0.3$ and $\omega=1$; so we keep the same frequency with the previous simulation by decrease the amplitude of the excitation, or equivalently, the energy input in the system. Judging from the FEP in Fig. 19, we make the theoretical prediction that by decreasing the energy input from a high to a sufficiently low level while keeping the frequency fixed, the dynamics should make a transition from the PB to the AB. Hence, the dynamics should change qualitatively, and from spatially extended become spatially confined. This is confirmed by the numerical simulations presented in Fig. 21, where we note that in this case no energy transmission occurs through the chain, but rather the input energy is confined in a region close to the left boundary where it is originally generated by the oscillating left rigid wall. Moreover, from the transient responses of system presented in Fig. 21a we note that after some initial transients the dynamics settles into a pattern of a standing wave with a decaying envelope; it follows that the response of the system becomes increasingly smaller away from the point of base excitation. This is in agreement with our remarks regarding the near-field motions that typically occur inside the ABs. We conclude that, contrary to the previous simulation, in this case the intrinsic dynamics of the granular chain does not enable the transmission of disturbances through the granular medium. In addition, from the snapshot plots in Fig. 21b we conclude that with increasing time the motion inside the AB settles into a near-linear decaying configuration where the beads nearest to the excitation have the greatest offsets from the trivial equilibrium, whereas the ones furthest have negligible offsets (this is also confirmed by the waveforms in Fig. 21a). This indicates that there is an effective pre-compression in the granular chain away from the excitation point. Therefore, the dynamics in the AB can be studied by adopting a weakly nonlinear approach. We may expand the response of each bead in Taylor series about its mean offset position and retain only the leading-order nonlinear terms. Then we should be able to derive analytical approximations to the spatially decaying waves inside the AZ depicted in Fig. 21a.

Comparing the temporal evolution of the total energy in the system (i.e., Figs. 20c and 21c) we note that for excitation frequency inside the $\mathrm{AB}$ the energy decays with time and reaches a zero steady-state value, whereas for excitation frequency inside the PB the energy reaches a steady state where it fluctuates about a non-zero mean value (actually, the computation of this mean value can help us to represent approximately the 

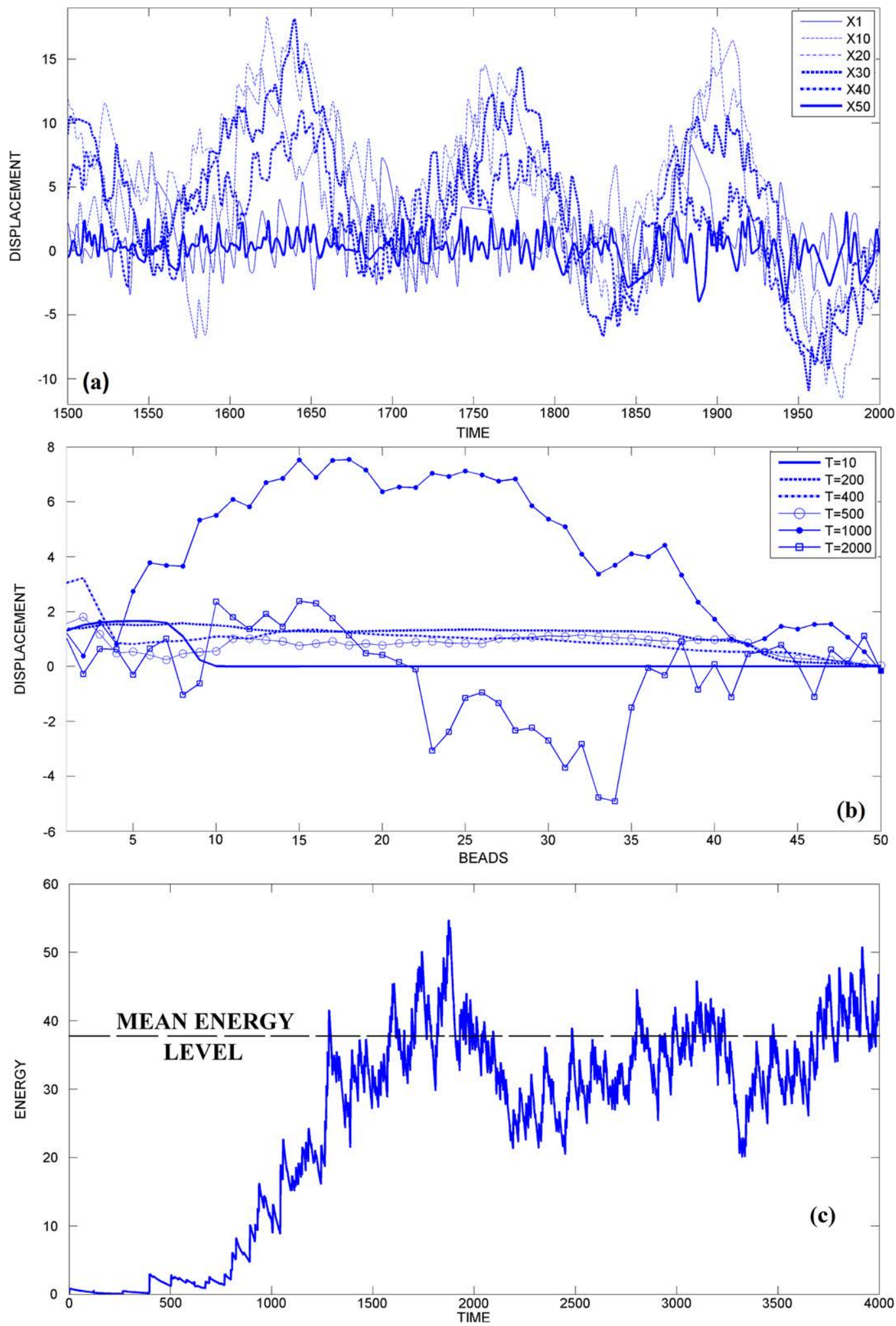

Fig. 20 Forced response of the 50-bead granular chain for harmonic wall excitation $y_{0}=1.5 \sin \tau$ : (a) transient responses of selected beads for $1500<\tau<2000$, (b) snapshots of chain deformation at selected time instants, (c) evolution of total energy in the chain 

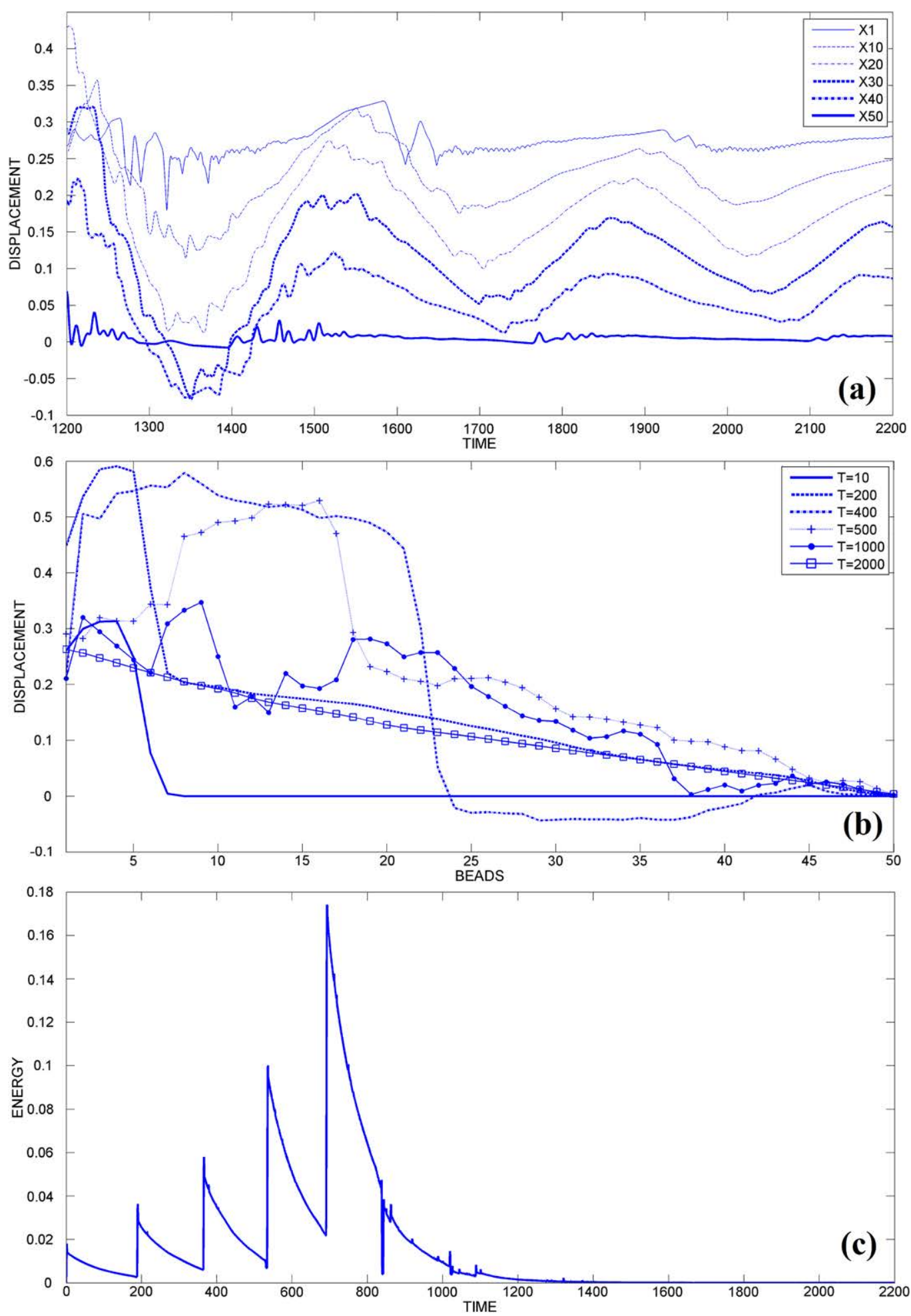

Fig. 21 Forced response of the 50-bead granular chain for harmonic wall excitation $y_{0}=0.3 \sin \tau$ : (a) transient responses of selected beads for $1000<\tau<2000$, (b) snapshots of chain deformation at selected time instants, (c) evolution of total energy in the chain 
response depicted in Fig. 20 in the FEP of Fig. 19a). This is a clear demonstration of the capacity of the intrinsic dynamics of the granular medium to attenuate energy inside the $\mathrm{AB}$ of the FEP.

We conclude that the results presented in this section indicate that the topological structure of the $\mathrm{PB}$ and $\mathrm{AB}$ of the frequency-energy plot affects significantly the narrowband-forced dynamics of the granular chain. Indeed, depending on the frequency-energy content of the excitation, the intrinsic dynamics of the medium either facilitates or hinders the spatial propagation of disturbances within the granular medium, allowing the propagation or dissipating certain frequency components. To extend this study to broadband (shock) excitation it will be necessary to add dissipative effects such as dry friction or plasticity to the Hertzian law interaction considered herein. We expect that such dissipative effects will reveal clearly the effect of the intrinsic dynamics on the broadband response of the granular medium. Moreover, it should be possible to identify transitions in the dissipative dynamics in the FEP, in an exactly similar way performed for transient smooth nonlinear dynamics of dissipative-coupled oscillators [17]. Such studies can be used in formulating predictive designs of this type of granular media as passive mitigators of shocks or other types of unwanted transient disturbances.

\section{Concluding remarks}

New types of NNMs in granular media have been studied in this work. These media have the intriguing feature of 'sonic vacuum,' that is, the corresponding velocity of sound in these media is zero due to the essentially nonlinear (nonlinearizable) Hertzian contact interactions between neighboring beads. Moreover, nonsmooth effects are added to the dynamics due to bead separation when in the absence of compressive forces between them. Though the traditional terminology of NNMs is restricted to smooth dynamical systems, the essentially nonlinear and non-smooth features of the dynamics of granular media force us to broaden the definition of NNMs to include time-periodic orbits that are not necessarily synchronous. In the case of granular systems, the non-synchronicity is caused by bead separation, which can lead to 'silent' regions in the bead dynamics, whereby one or more beads become motionless for a finite period of time at an offset position from the zero equilibrium.

Clearly, the most interesting of the NNMs of the homogeneous granular systems studied in this work is the in-phase NNM, which to the authors' knowledge has not been studied before in the scientific literature. This mode can be realized only when the initial state of the beads of the granular medium have prescribed gaps between them, i.e., for zero initial velocities, the initial bead displacements should be such that no bead is in touch with another bead or with the walls, except for one of the end beads. The in-phase NNM resembles a traveling wave propagating backward and forward through the finite granular chain, and hence it was labeled as pseudo-wave. In addition, it can only exist in the finite chain, since in the limit of infinite number of beads the gaps between beads (required for the excitation of this mode) tend to zero. On the contrary, the solitary wave studied by Nesterenko [9] does not involve bead separation, so it can be studied by an analysis based on continuum approximation of the strongly nonlinear equations of motion.

The significance of the in-phase (pseudo-wave) NNM and the out-of-phase NNM of the granular chain is that they form the boundaries of the band in the frequency-energy plane within which all other NNMs are realized. It follows that as the number of beads tends to infinity this region forms the propagation band (PB) in the frequency-energy plot (FEP), whereas the complementary region forms the attenuation band $(\mathrm{AB})$. Motions inside the PB transfer energy through the medium and are spatially extended, whereas the corresponding motions inside the $\mathrm{AB}$ are near-field solutions. Hence, the topologies of these bands in the frequency-energy plane affect significantly the forced dynamics of the granular medium. Indeed, frequency components inside the PB propagate unattenuated whereas components inside the $\mathrm{AB}$ are damped by the intrinsic dynamics of the medium. In the event of application of a high frequency impulsive/shock loading, the frequency components in the $\mathrm{PB}$ are transmitted along the chain, whereas the frequency components in the $\mathrm{AB}$ are attenuated. As discussed previously, when the system is harmonically excited at high frequency, the system settles down to a localized mode and the high frequency signal is completely attenuated. This result, which is confirmed by direct numerical simulations reported in this work, has considerable practical significance 
in designing this type of strongly nonlinear granular media as passive mitigators of unwanted disturbances.

Acknowledgements This work was funded by MURI grant US ARO W911NF-09-1-0436, Dr. David Stepp is the grant monitor. The authors would like to thank Professor Leonid I. Manevitch of the Institute of Chemical Physics, Russian Academy of Science, and Professors John Lambros and Philippe Geubelle of the University of Illinois at Urbana-Champaign for valuable discussions and suggestions.

\section{References}

1. Lyapunov, A.: Probleme generale de la stabilité du mouvement. Ann. Fas. Sci. Toulouse 9, 203-474 (1907)

2. Pak, C.H., Rosenberg, R.M.: On the existence of normal mode vibrations in nonlinear systems. Q. Appl. Math. 26, 403-416 (1968)

3. Rand, R.H.: Nonlinear normal modes in two-degrees-offreedom systems. J. Appl. Mech. 38, 561 (1971)

4. Rosenberg, R.M.: The normal modes of nonlinear n-degrees-of-freedom systems. J. Appl. Mech. 30, 7-14 (1962)

5. Rosenberg, R.M., Kuo, J.: Nonsimilar normal mode vibrations of nonlinear systems having two degrees of freedom. J. Appl. Mech. 31, 283-290 (1964)

6. Rosenberg, R.M.: On nonlinear vibrations of systems with many degrees of freedom. Adv. Appl. Mech. 9, 155-242 (1966)

7. Vakakis, A.F., Manevitch, L.I., Mikhlin, Yu., Pilipchuck, V., Zevin, A.A.: Normal Modes and Localization in Nonlinear Systems. Wiley, New York (1996)

8. Vakakis, A.F.: Analysis and identification of linear and nonlinear normal modes in vibrating systems, $\mathrm{Ph} . \mathrm{D}$. Thesis, California Institute of Technology, Pasadena, California (1990)

9. Nesterenko, V.F.: Dynamics of Heterogeneous Materials. Springer, New York (2001)

10. Daraio, C., Nesterenko, V.F., Herbold, E.B., Jin, S.: Tunability of solitary wave properties in one-dimensional strongly nonlinear phononic crystals. Phys. Rev. E 73, 026610 (2006)

11. Daraio, C., Nesterenko, V.F., Herbold, E.B., Jin, S.: Strongly nonlinear waves in a chain of Teflon beads. Phys. Rev. E 72, 016603 (2005)

12. Sen, S., Hong, J., Bang, J., Avalos, E., Doney, R.: Solitary waves in the granular chain. Phys. Rep. 462, 21 (2008)

13. Nesterenko, V.F., Daraio, C., Herbold, E.B., Jin, S.: Anomalous wave reflection at the interface of two strongly nonlinear granular media. Phys. Rev. Lett. 95, 158702 (2005)
14. Herbold, E.B., Kim, J., Nesterenko, V.F., Wang, S.Y., Daraio, C.: Pulse propagation in a linear and nonlinear diatomic periodic chain: effects of acoustic frequency bandgap. Acta Mech. 205, 85 (2009)

15. Abramowitz, M., Stegun, I.A.: Handbook of Mathematical Functions. Dover, New York (1972)

16. Starosvetsky, Y., Vakakis, A.F.: Traveling waves and localized modes in one-dimensional homogeneous granular chains with no pre-compression. Phys. Rev. E 82, 026603 (2009)

17. Peeters, M., Viguié, R., Sérandour, G., Kerschen, G., Golinval, J.C.: Nonlinear normal modes. Part II. Toward a practical computation using numerical continuation. Mech. Syst. Signal Process. 23, 195-216 (2009)

18. Theocharis, G., Kavousanakis, M., Kevrekidis, P.G., Daraio, C., Porter, M.A., Kevrekidis, I.G.: Localized breathing modes in granular crystals with defects. arXiv:0906.4094v1 [nlin.PS] (2009)

19. Boechler, N., Daraio, C.: An experimental investigation of acoustic band gaps and localization in granular elastic chains. In: Proceedings of the ASME, 2009 International Design Engineering Technical Conferences \& Biennial Conference on Mechanical Vibration and Noise IDETC/CIE Aug. 30-Sept. 2, 2009, San Diego, California

20. Mead, D.J.: Wave propagation and natural modes in periodic systems. I. Monocoupled systems. J. Sound Vib. 40, 1-18 (1975)

21. Vakakis, A.F., Cetinkaya, C.: Dispersion of stress waves in one-dimensional semi-infinite, weakly coupled layered systems. Int. J. Solids Struct. 33(28), 4195-4213 (1996)

22. Norris, A.W.: Waves in periodically layered media: a comparison of two theories. SIAM J. Appl. Math. 53, 11951209 (1993)

23. Velo, A.P., Gazonas, G.A., Ameya, T.: z-Transform methods for the optimal design of one-dimensional layered elastic media. SIAM J. Appl. Math. 70(3), 762-788 (2009)

24. Vakakis, A.F., King, M.E.: Nonlinear wave transmission in a monocoupled elastic periodic system. J. Acoust. Soc. Am. 98(3), 1534-1546 (1995)

25. Cetinkaya, C., Vakakis, A.F., El-Raheb, M.: Axisymmetric elastic waves in weakly coupled layered media of infinite radial extent. J. Sound Vib. 182(2), 283-302 (1995)

26. Vakakis, A.F., King, M.E.: Resonant oscillations of a weakly coupled, nonlinear layered system. Acta Mech. 128, 59-80 (1998)

27. Jiang, D., Pierre, C., Shaw, S.W.: Large-amplitude nonlinear normal modes of piecewise linear systems. J. Sound Vib. 272(3-5), 869-891 (2004)

28. Chen, S., Shaw, S.W.: Normal modes for piecewise linear vibratory systems. Nonlinear Dyn. 10, 135-163 (1996)

29. Zuo, L., Curnier, A.: Non-linear real and complex modes of conewise linear systems. J. Sound Vib. 174, 289-313 (1994) 